\title{
Functional Anatomy of the Second Visual Area (V2) in the Macaque
}

\author{
Roger B. H. Tootell and Susan L. Hamilton \\ Department of Psychology, University of California, Berkeley, Berkeley, California 94720
}

To study the functional organization of secondary visual cortex (V2) in the primate, ${ }^{14} \mathrm{C}$-2-deoxy-d-glucose (DG) was injected while macaque monkeys were shown specific visual stimuli. Wherever possible, patterns of DG uptake were compared with the position of dark and light cytochrome oxidase (cytox) stripes (Tootell et al., 1983). Often, the DG effects of 2 different stimuli were compared in the same hemisphere to eliminate ambiguities inherent in between-animal comparisons. Data were obtained from a large number of animals in conjunction with related DG studies in area $\mathrm{V} 1$ (primary visual or striate cortex).

The following conclusions were reached: (1) In some macaque monkeys, dark cytox stripes were faint or absent. Although this could conceivably be due to poor staining technique, some evidence suggests that the lack of enzyme stripe pattern is real. In all animals, including those that showed poor or no cytox staining evidence for stripes, the functional architecture revealed by the DG was consistently present and robust. (2) Uniform gray stimuli produce a relatively uniform pattern and minimal stimulus-related DG uptake. (3) Eye movements per se produce some uptake in the V2 stripes. (4) Very generalized visual stimulation conditions (e.g., binocular stimulation with a grating of varied orientation and varied spatial frequency) produce a pattern of uptake that is greatest in both sets of dark cytox stripes and lighter in the light cytochrome stripes. (5) In both the DG and cytox results, the V2 "stripes" are more accurately described as stripe-shaped collections of patches. (6) In almost all cases, DG patterns were columnar in shape, extending trom white matter to cortical surface. The boundaries of the columns were most sharply defined, and the contrast was highest, in layers $3 B / 4$, becoming slightly more blurry and lower in contrast in other layers. Laminar differences between DG patterns in V2 were almost negligible, compared with the profound laminar differences in macaque V1. (7) There is no DG evidence for, and much against, the possibility of an ocular dominance architecture in V2. (8) There are orientation columns in macaque V2. DG-labeled orientation columns are spaced further apart than those in $V 1$, by a factor of

\footnotetext{
Received Sept. 26, 1988; revised Dec. 13, 1988; accepted Dec. 14, 1988.

Special thanks are extended to Martin S. Silverman, who collaborated on some of the preliminary data; to Gene Switkes, who programmed most of the visual stimuli; and to Russell De Valois, who graciously allowed us to use some of his equipment and supplies. This work was supported by United States Public Health Services Grants EY-00014, EY-02050, and EY-07980, and National Science Foundation Grant BNS82-02275.

Correspondence should be addressed to Roger B. H. Tootell, Department of Neurobiolngy, Harvard Medical School, 220 I ongwood Avenue, Boston, MA 02115

Copyright (C) 1989 Society for Neuroscience $0270-6474 / 89 / 082620-25 \$ 02.00 / 0$
}

about 1.6, but the columns are not correspondingly wider. (9) Spatially diffuse variations in color produce high uptake confined, at least largely, to the thin cytox stripes. (10) There is evidence for spatially antagonistic color surrounds in color cells in the thin stripes. (11) In general, achromatic gratings of low luminance contrast $(\sim 8 \%)$ produce faint-to-medium DG uptake confined in thick V2 stripes, and little or no stimulus-driven DG uptake outside the thick V2 stripes. Since such stimuli stimulate magnocellular but not parvocellular LGN cells, this suggests that $\mathbf{V} 2$ receives input mainly from parvocellular rather than magnocellular channels, except for the magnocellular-derived input to the thick V2 stripes. (12) Variations in the spatial frequency of achromatic, sinusoidal gratings produce corresponding variations in the DG patterns from V2 (see also Tootell et al., 1983). Binocular stimulation with a grating of low $(\sim 1.5 \mathrm{c} / \mathrm{deg})$ spatial frequency at all orientations produces high DG uptake on every dark cytox stripe. Comparable stimulation with a high $(\sim 6.5 \mathrm{c} / \mathrm{deg})$ spatial frequency produces isolated columns of high uptake, often aligned along the light cytox interstripes.

As mammalian cortex continues to be explored, it has become increasingly evident that cells with common functional properties are very often grouped together anatomically. In addition to the classical examples of ocular dominance and orientation columns in primary visual cortex (Hubel and Wiesel, 1962, 1968), there is more recent electrophysiological evidence for directional columns in the middle temporal area (MT) (Albright et al., 1984); color, end-stopped and disparity groupings (probably columnar) in visual area V2 (DeYoe and Van Essen, 1985; Hubel and Livingstone, 1985, 1987); and cells grouped according to color and receptive field differences in primary visual cortex (Livingstone and Hubel, 1984; Tootell et al., 1988c, e).

Electrophysiological mapping data is essential for getting a fine-grained impression of the cellular responses. However, for mapping per se it is tedious and subject to sampling limitations. Cortical mapping studies have been greatly extended by the use of the DG technique (e.g., Kennedy et al., 1975; Hubel et al., 1978; Hendrickson and Wilson, 1979; Humphrey et al., 1980; Horton and Hubel, 1981; Schoppmann and Stryker, 1981; Tootell et al., 1982, 1983, 1988a-e) and more recently by optical techniques (Orbach et al., 1985; Blasdel and Salama, 1986; Grinvald et al., 1986).

Macaque visual area $\mathrm{V} 2$ has recently become ripe for a thorough analysis of functional organization. Though area V2 is the major projection region of the primary visual cortex, and though it is almost as large as the primary visual cortex, the area was comparatively unexplored until recently.

This relative neglect has been partially rectified since the dem- 
onstration of cytochrome oxidase (cytox) stripes in V2 (Livingstone and Hubel, 1983; Tootell et al., 1983; Horton, 1984). In $\mathrm{V} 2$ tissue that has been stained for the mitochondrial enzyme cytox, dark staining stripes alternate with parallel stripes of lighter staining running perpendicular to the $\mathrm{V} 1-\mathrm{V} 2$ border. In the squirrel monkey, the dark stripes clearly alternate between thin and thick, with lighter stripe-shaped regions of staining between them (Tootell et al., 1983). This enzyme distinction is less evident in the macaque, but even in the macaque there is a strict functional organization of cells into "thin" and "thick" stripes (e.g., DeYoe and Van Essen, 1985; Hubel and Livingstone, 1987; and also present results). The blobs in layers $2+3$ of $V 1$ project to the dark thin stripes in $\mathrm{V} 2$, and the pale stripes in $\mathrm{V} 2$ receive input from the pale interblob regions of V1 (Livingstone and Hubcl, 1983). Laycr 4B projects to the dark thick stripes (Livingstone and Hube!, 1987). The thick stripes project to cortical area $\mathrm{MT}$, and the thin and interstripe regions project to cortical area V4 (DeYoe and Van Essen, 1985; Shipp and Zeki, 1985). According to single-unit reports, cells in the thin stripes are color-coded, cells in the thick stripes are disparity-specific, and cells in the lighter interstripe regions are end-stopped (DeYoe and Van Essen, 1985; Hubel and Livingstone, 1985, 1987). From the segregated projection of the magnocellular and parvocellular LGN layers through striate cortex (Lund, 1973; Lund and Boothe, 1975; Maunsell, 1987; Tootell et al., 1988d), it appears that much of the information that projects to the thin and interstripe areas of $\mathrm{V} 2$ is derived from the parvocellular layers, and much of the input to the thick stripes is routed through the magnocellular LGN layers (e.g., Livingstone and Hubel, 1983; Tootell et al., 1988d). It has been suggested that the connections of the V2 stripes are different in Old and New World monkeys (Krubitzer and Kaas, 1987), but this issue has not yet been fully resolved.

From all this we can see the broad outlines of an understanding of V2 functional organization, but many questions remain. In the present study, we used ${ }^{14} \mathrm{C}$-2-deoxy- $d$-glucose (DG) to label the pattern of functional activity in response to specific visual stimuli to begin to answer some of these questions.

\section{Materials and Methods}

Most of the material in the present study was generated originally in a study of the functional organization of primary visual cortex, area V1. Full details of the methods can be found in Tootell et al. (1988a). We used 59 macaque monkeys ( $M$. fascicularis, $M$. arctoides, $M$. nemestrina, $M$. assamensis, or $M$. radiata). The animals were implanted with plastic head pedestals about a week prior to the main DG experiment; this eliminated the need for ear bars during the later experiment. During the main experiment, monkeys were anesthesized, paralyzed, and prepared as for electrophysiological recording. An intravenous catheter was used for the injection of various drugs, and the monkeys were artificially respired through endotracheal tubes. Either one or both eyes were focused on the center of the stimulus screen. In about half of the cases, multiunit activity recorded from foveal striate cortex was used both to locate the foveal projection in the visual field and also as a measure of binocular correspondence. Visual stimuli were presented on a Tektronix color monitor driven by a Lexidata image processor. Often 2 different visual stimuli were used, positioned on either side of the horizontal meridian. These "split-field" stimuli were designed so that the DG effects of the 2 stimuli could be compared within a given hemisphere in the same tissue section. The visual stimulus parameters that were manipulated included color, orientation, contrast, spatial frequency, direction, ocular dominance, binocular disparity, and retinotopic position. Specific details of each visual stimulus are given case-by-case under Results and in Table 1.

When the optics were arranged and the eye(s) converged on the center of the screen, DG $(12.5-50 \mu \mathrm{Ci} / \mathrm{kg})$ was injected while the monkey viewed the stimulus. Protracted injections (up to $15 \mathrm{~min}$ ) were used in order to prevent inadvertent stimulus biases in the patterns of DG uptake. Generally, a single visual stimulus was presented for $60 \mathrm{sec}$, followed by the next stimulus in a randomized series of all stimuli used in that set of conditions. After 45-60 min, monkeys were overdosed with sodium pentobarbital $(50-75 \mathrm{mg} / \mathrm{kg})$ and transcardially perfused with a $13 \%$ sucrose, phosphate-buffered solution, a light formalin fixation, followed by another rinse with the sucrose-phosphate-buffered solution. The brain was removed from the skull, and the operculum (including the central $10^{\circ}$ representation of area V2) was dissected free from the rest of the brain.

In most cases, the tissue was flattened parallel with the lateral surface of the operculum, which is primarily striate cortex (Tootell and Silverman, 1985). In 8 cases, the tissue was flattened parallel to the surface of area V2, on the posterior bank of the lunate sulcus and on the dorsal bank of the inferior occipital sulcus. Tissue was cut on a cryostat parallel with the flattened plane. In the few cases where area V2 was flattened instead of V1, the plane of section runs near-parallel to the cortical layers of V2. In the majority of cases, the central half of area V1 was flattened so that the sectioning plane runs slightly oblique to the V2 layers at an angle near $5^{\circ}-10^{\circ}$. Autoradiographs from the tissue sections were exposed at $-70^{\circ} \mathrm{C}$. After autoradiography, the tissue was stained for cytox (Tootell et al., 1985, 1988a; Silverman and Tootell, 1987).

Stripe staining. In the squirrel monkey, cytox stripes show up quite robustly in cortical area V2 in every case we have analyzed (e.g., Tootell et al., 1983). In the macaque monkey, the situation is different. In some (perhaps half) of the tissue we examined from macaque V2, cytox stripes were either very faint or absent.

For several reasons we believe the lack of V2 stripes in the macaque is not due to poor staining technology. (1) First, faint V2 stripes occurred even when the V2 tissue was reacted in the same solutions with tissue from striate cortex (V1) in which the various laminae and blobs were clearly visible. No such variability was seen in the staining of the V1 blobs. (2) In all of the V2 cytox tissue, laminar boundaries were clearly visible. (3) In a number of cases from a related study, the full extent of area V2 was flattened and stained (e.g., Tootell and Silverman, 1985). In this tissue there was variability not only between different individuals but within a given animal as well: some parts of $\mathrm{V} 2$ show stripes, and other parts do not (see figure 4 from Tootell and Silverman, 1985). A similar result was obtained from human V2, another Old World primate (unpublished observations). (4) In one direct test, we reacted every other V2 section for cytox in 2 conditions: (a) immediately after sectioning and (b) after desiccation and 9 weeks of autoradiographic exposure at $-70^{\circ} \mathrm{C}$. No difference in the staining intensity of the $\mathrm{V} 2$ stripes was seen in the 2 conditions.

There are at least 3 possible interpretations in the face of such variability in V2 stripes: (1) The stripes do not exist in V2 some of the time; (2) the enzyme differences that make the V2 stripes visible do not exist some of the time; or (3) the cytox stripes exist but are for some reason more labile than the V1 blob cytox differences and, hence, especially subject to histological compromise. Based on many lines of evidence, we favor the second possibility: the functional (and presumably the connectional) substrates of the $\mathrm{V} 2$ stripes always exist, but the enzyme differences that have often been used to define the $\mathrm{V} 2$ stripes are present more variably. However, we cannot absolutely rule out the possibility that the lack of stripe staining is an artifact of poor staining technology.

At any rate, we found that robust DG patterns were always produced by appropriate visual stimulation, even when the cytox staining showed little evidence of V2 stripes. Figure 1 shows some of this evidence. Figure $1, A-C$ presents autoradiographs from V2, from an animal visually stimulated with spatially diffuse variations in color (case 6 ; see Table 1). Further details of the visual stimulus are given below, in the color section; for the moment they are irrelevant. Figure $1, A-C$ was taken from different layers of the same tissue block. The portion of V2 illustrated in Figure 1, $A-C$ extends from the representation of the fovea to about $8^{\circ}$ eccentricity. Clear stripes of high DG uptake are visible running through the autoradiographs, perpendicular to the V1-V2 border. (This border lies near the top edge of the tissue in Fig. 1, $A-C$.) These stripes turn out to be coextensive with the thin cytox stripes, in animals that do show cytox stripes.

Figure $1 D$ shows the same tissue section that produced Figure $1 B$, after staining for cytox. The section was stained in a reaction solution that revealed V1 blobs quite well (not shown). The dark and light stripes simply do not exist, or are very faint, in this and much other cytoxstained tissue from macaque $\mathrm{V} 2$. 


\begin{tabular}{|c|c|c|c|}
\hline Case & Species & $\begin{array}{l}\text { Viewing } \\
\text { conditions }\end{array}$ & Stimulus \\
\hline 1 & Fas & Bin & Spatially diffuse red-green flicker \\
\hline 2 & Fas & Bin & Spatially diffuse black-white flicker \\
\hline 3 & Nem & Bin & Spatially diffuse red-gray flicker \\
\hline 4 & Fas & Bin & $\begin{array}{l}\text { Split-field ( } 2 \text { sector), spatially diffuse red-gray vs luminance varying } \\
\text { flicker }\end{array}$ \\
\hline 5 & Arc & Bin & Spatially diffuse blue-gray flicker \\
\hline 6 & Fas & Bin & Spatially diffuse red-gray flicker \\
\hline 7 & Fas & Bin & Spatially diffuse black-white flicker \\
\hline 8 & Fas & Bin & Horizontal, var. sp. freq. square-wave grating \\
\hline 9 & Arc & Bin & Ring-and-ray stimulus (checks) \\
\hline 10 & Fas & Bin & Diffuse gray screen (no flicker) \\
\hline 11 & Arc & Mon & Ring-and-ray stimulus (solid) \\
\hline 12 & Arc & Bin & Var, orientation, var. sp. freq., var. color, square-wave grating \\
\hline 13 & Arc & Mon & Ring-and-ray stimulus (checks) \\
\hline 14 & Arc & Bin & Vertical, var. sp. freq. square-wave grating (var. disparity) \\
\hline 15 & Arc & Mon & Var. orientation, var. sp. freq. square-wave grating \\
\hline 16 & Fas & Bin & Unparalyzed. unrestrained in the dark \\
\hline 17 & Fas & Mon & Var. orientation, var. sp. freq., red-gray square wave grating \\
\hline 18 & Fas & Mon & Ring-and-ray stimulus (checks) \\
\hline 19 & Fas & Bin & Var. orientation, $6.5 \mathrm{c} / \mathrm{deg}$ sinusoidal grating (var. disparity) \\
\hline 20 & Fas & Bin & Var. orientation, $1 \mathrm{c} / \mathrm{deg}$ sinusoidal grating (var. disparity) \\
\hline 21 & Fas & Mon & Horizontal, $6.5 \mathrm{c} / \mathrm{deg}$ sinusoidal grating \\
\hline 22 & Fas & Mon & $\begin{array}{l}\text { Split-field ( } 2 \text { sector), one-vs-var. orientation, var. sp. freq. sinusoidal } \\
\text { grating }\end{array}$ \\
\hline 23 & Arc & Mon & $\begin{array}{l}\text { Split-field ( } 2 \text { sector), color-vs-luminance varying sinusoidal grating, } \\
\text { var. orientation, var. sp. freq. (with blank-out rings) }\end{array}$ \\
\hline 24 & Fas & Bin & Split-field (2 sector), $7 \mathrm{c} /$ deg var. sp. freq. sinusoidal grating \\
\hline 25 & Fas & Bin & $\begin{array}{l}\text { Split-field ( } 8 \text { sector) spatially diffuse color-gray flicker (red-vs-green- } \\
\text { vs-ycllow-vs-blue/gray } \times 2 \text { ) }\end{array}$ \\
\hline 26 & Fas & Mon & Vertical mcridian, var. oricntation, var. sp. freq. square-wave grating \\
\hline 27 & Fas & Mon & $\begin{array}{l}\text { Split-field ( } 4 \text { sector) } 6.5 \text {-vs-1 c/deg color-vs-luminance varying, } \\
\text { sinusoidal grating }\end{array}$ \\
\hline 28 & Fas & Bin & Vertical, $6.6 \mathrm{c} / \mathrm{deg}$ sinusoidal grating (var. disparity) \\
\hline 29 & Arc & Mon & $\begin{array}{l}\text { Split-field ( } 4 \text { sector) } 6.5 \text {-vs- } 1 \mathrm{c} / \mathrm{dcg} \text {, color-vs-luminance-varying } \\
\text { sinusoidal grating, var, orientation }\end{array}$ \\
\hline 30 & Arc & Mon & $\begin{array}{l}\text { Split-field ( } 2 \text { sector) } 7-v s-1 \mathrm{c} / \text { horizontal sp. freq. sinusoidal grating } \\
\text { (with blank-out rings) }\end{array}$ \\
\hline 31 & Fas & Bin & Horizontal, $7 \mathrm{c} / \mathrm{deg}$ sp. freq. sinusoidal grating \\
\hline 32 & Fas & Bin & Vertical, $0.9 \mathrm{c} / \mathrm{deg}$ sp. freq. sinusoidal grating (var. disparity) \\
\hline 33 & Fas & Mon & $\begin{array}{l}\text { Split-field ( } 3 \times 2 \text { sector), spatially-diffuse color-gray flicker (red-vs- } \\
\text { green-vs-blue/gray), equal saturation vs equal purity }\end{array}$ \\
\hline 34 & Ass & Mon & $\begin{array}{l}\text { Split-field ( } 4 \text { sector) } 4.1-\mathrm{vs}-0.7 \mathrm{c} / \mathrm{deg} \text {, color-vs-luminance-varying } \\
\text { sinusoidal grating }\end{array}$ \\
\hline 35 & $\operatorname{Rad}$ & Mon & $\begin{array}{l}\text { Split-field ( } 4 \text { sector) } 8 \text {-vs- } 18 \text {-vs- } 40-v s-100 \% \text { contrast square-wave } \\
\text { grating, horizontal, var. sp. freq. }\end{array}$ \\
\hline 36 & Ass & Mon & $\begin{array}{l}\text { Split-field ( } 4 \text { sector) } 4.1-v s-0.7 \mathrm{c} / \mathrm{deg} \text { color-vs-luminance-varying } \\
\text { sinusoidal grating, var. orientation }\end{array}$ \\
\hline 37 & Ass & Bin & Var. orientation, var. sp. freq. square-wave grating (var. disparity) \\
\hline 38 & Fas & Mon & $\begin{array}{l}\text { Split-field ( } 4 \text { sector) red-vs-blue-vs-green/gray-vs-black/white, } 2.7 \\
\text { c/deg sinusoidal grating, var. oricntation (with blank-out rings) }\end{array}$ \\
\hline 39 & Ass & Bin & $\begin{array}{l}\text { Split-field ( } 2 \text { sector) } 4.4-v s-0.7 \mathrm{c} / \mathrm{dcg} \text { sinusoidal grating, var. } \\
\text { orientation }\end{array}$ \\
\hline 40 & Nem & Bin & Split-field ( 2 sector) 7 -vs-1 c/deg sinusoidal grating, var. orientation \\
\hline 41 & Arc & Mon & $\begin{array}{l}\text { Split-field (6 sector) spatially diffuse color-gray flicker (red-vs-green- } \\
\text { vs-blue/gray) equal saturation vs equal purity }\end{array}$ \\
\hline 42 & Fas & Bin & Var. orientation, var. sp. freq. square-wave grating \\
\hline 43 & Ass & Mon & $\begin{array}{l}\text { Split-field ( } 4 \text { sector) } 8 \text {-vs- } 18 \text {-vs- } 38 \text {-vs- } 100 \% \text { contrast, var. sp. freq. } \\
\text { square-wave grating, oblique orientation }\end{array}$ \\
\hline
\end{tabular}




\begin{tabular}{|c|c|c|c|}
\hline Case & Species & $\begin{array}{l}\text { Vicwing } \\
\text { conditions }\end{array}$ & Stimulus \\
\hline 44 & Arc & Mon & $\begin{array}{l}\text { Split-field ( } 2 \text { sector) } 4.8-v s-0.7 \mathrm{c} / \mathrm{deg} \text { sp. freq. square-wave grating, } \\
\text { oblique orientation (with blank-out rings) }\end{array}$ \\
\hline 45 & Ass & Bin & $8 \%$ contrast, var. orientation, var. sp. freq. square-wave grating \\
\hline 46 & Ass & Bin & $\begin{array}{l}\text { Split-field ( } 2 \text { sector) } 180^{\circ} \text {-vs- } 360^{\circ} \text { directions, var. orientation, var. sp. } \\
\text { freq. square-wave grating }\end{array}$ \\
\hline 47 & Fas & Bin & Random dot pattern, one direction \\
\hline 48 & Arc & Mon & $\begin{array}{l}\text { Split-field ( } 4 \text { sector) } 0.1-v s-2-4.4 \mathrm{c} / \mathrm{deg} \text { red-gray vs. } 0.1 \mathrm{c} / \mathrm{deg} \text { black- } \\
\text { white sinusoidal grating, var. orientation }\end{array}$ \\
\hline 49 & Arc & Bin & $\begin{array}{l}\text { Split-field ( } 2 \text { sector) } 4.4-v s-1 \mathrm{c} / \mathrm{deg} \text { sinusoidal grating, var. } \\
\text { orientation }\end{array}$ \\
\hline 50 & Arc & Bin/ & $\begin{array}{l}\text { Split-field ( } 2 \text { sector) binocular vs monocular, var. sp. freq. sinusoidal } \\
\text { grating, var. orientation }\end{array}$ \\
\hline 51 & Fas & Bin & $\begin{array}{l}7.5 \mathrm{c} / \text { deg square-wave grating, var. orientation (hand-held, } \\
\text { unparalyzed) }\end{array}$ \\
\hline 52 & Nem & Mon & Horizontal, $0.5 \mathrm{c} / \mathrm{deg}$ stationary, counterphased square-wave grating \\
\hline 53 & Nem & Mon & $\begin{array}{l}\text { Split-field ( } 2 \text { sector) red-gray-vs-red-black, } 6.5 \mathrm{c} / \mathrm{deg} \text { square-wave } \\
\text { grating, var. orientation }\end{array}$ \\
\hline 54 & Fas & Bin & $6.5 \mathrm{c} / \mathrm{deg}$ red-gray squarc-wave grating \\
\hline 55 & Nem & Bin & $\begin{array}{l}\text { Split-field ( } 2 \text { sector) } 6.5-\mathrm{vs}-1.5 \mathrm{c} / \mathrm{deg} \text { sinusoidal grating, var. } \\
\text { orientation }\end{array}$ \\
\hline 56 & Fas & Bin & Random dot pattern, var. directions \\
\hline
\end{tabular}

The species, viewing conditions, and stimulus parameters are described for each case. Five different species of macaques were used: $M$. fascicularis (Fas), $M$. nemestrina (Nem), $M$. arctoides (Arc), $M$. assamensis (Ass), and $M$. radiata (Rad). The viewing conditions were either monocular (Mon) or binocular (Bin) except in a specialized case ( $\# 50$ ). Unless specified otherwise, the stimuli were achromatic and the contrast was high $(70-100 \%)$. When color-varying stimuli were used, the colors were all equated for luminance. Abbreviations: var., variable; sp. freq., spatial frequency.

Such a discrepancy between cytox and DG periodicities was common in $\mathrm{V} 2$ but was never seen in V1. Where such a discrepancy occurred in $\mathrm{V} 2$, it was always the case that periodicities occurred in the DG but not the cytox results. The converse never occurred, except when the visual stimulus was known to be ineffective in producing DG uptake (c.g., Fig. $2 A$ below). At the very least, then, we can conclude that DG plus appropriate visual stimulation is one method that works well for defining the position of the $\mathrm{V} 2$ stripes in the macaque.

\section{Results}

\section{Baseline DG tests}

In all of our material, both the stripes and the patches that make them up are most clearly defined and highest in contrast in layers $3 \mathrm{~B} / 4$ but extend through the full extent of the gray matter (compare Fig. $1, A-C$ ). The DG patterns in V2 (and those in all other known extrastriate areas) show much less laminar variation than most of those in striate cortex; thus, in some ways the DG patterns in extrastriate cortex are more truly "columnar" than those in striate cortex. When demonstrated by cytox staining, the V2 stripes are also most clear in layers $3 \mathrm{~B} / 4$.

In striate cortex, there is very little difference between uptake in the blobs and in interblobs when striate cortex is visually unstimulated or when it is stimulated ineffectively. In such cases there is little uptake in blobs or interblobs. Since so much of the input to V2 is derived from the blobs or interblobs, one might expect an analogous result in V2.

In order to test this idea we examined DG results from a paralyzed, anesthesized animal (case 10) that had viewed a diffuse gray screen $(25 \mathrm{ft} \mathrm{L}$ ) during the time of DG infusion (see Fig. $2 A$ ). In this and most subsequent figures, the autoradiographs arc taken from area $\mathrm{V} 2$, from a representation of the fovea to about $8^{\circ}$ eccentricity, from sections cut near-parallel to the cortical layers. Such a diffusc gray stimulus produces essen- tially no uptake in $\mathrm{Vl}$, nor any obvious uptake in the major striate recipient area, V2 (see Fig. $2 A$ ). Here, the effect of the stimulus on levels of DG uptake could be assessed by comparing the visual field representation corresponding to regions inside and outside the stimulus borders.

We also wondered whether the presence of eye movements per se would have an effect on DG uptake. This is an important control case, because all of our other DG results were taken from animals in which eye movements were eliminated by pharmacologically paralyzing the cyes. To test this we injected DG into an animal (case 16) that was unparalyzed and unanesthesized (thus allowing eyc movements) but was kept in the dark to eliminate purely visual input. The results of this case are shown in Figure $2 B$. The pattern of uptake shows more variation than that in Figure $2 A$, and the regions of faintly higher DG uptake are, in fact, coincident with the dark cytox stripes. A similar result has been obtained in one other monkey treated identically in another study. Thus, there is DG evidence that (some) neurons in the dark cytox stripes are activated by the presence of eye movements. It would be very interesting to know what types of eye movements (saccades, slow pursuit, etc.) are reflected by the DG patterns, and what cells are involved.

Another case (case 59, not illustrated) supports the counterargument here-that uptake in the eye movement control case, while different, is not all that different. This animal was unparalyzed and unanesthesized, and its head was restrained so that the animal viewed a high spatial frequency grating during the period of DG uptake. In unparalyzed, lightly anesthesized animals such a stimulus produces high DG uptake in the interblob regions of striate cortex, and in the unparalyzed, unanesthesized case, we obtained a similar result (Tootell et al., 1988a). In area $\mathrm{V} 2$, both paralyzed and unparalyzed cases produced the same 


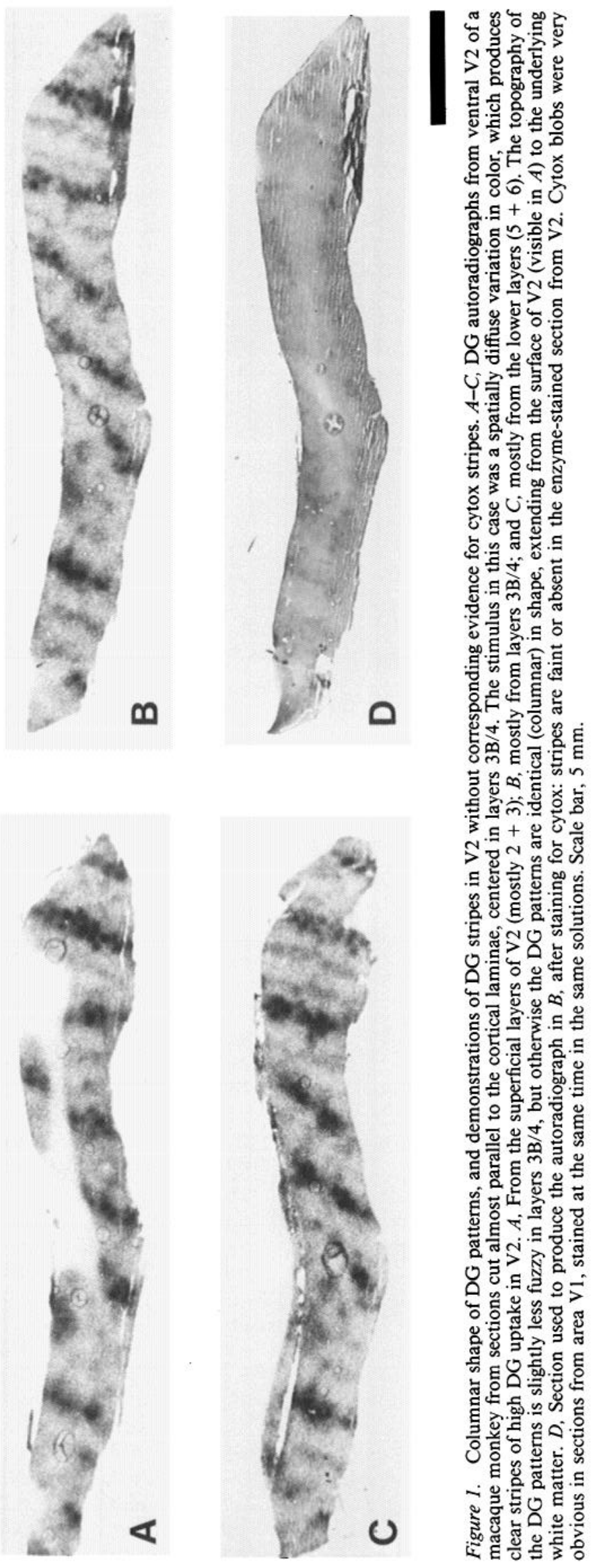




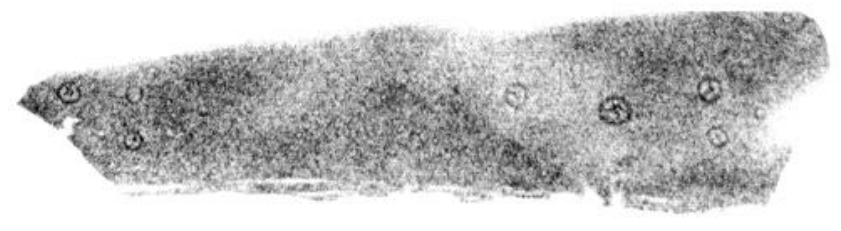

A

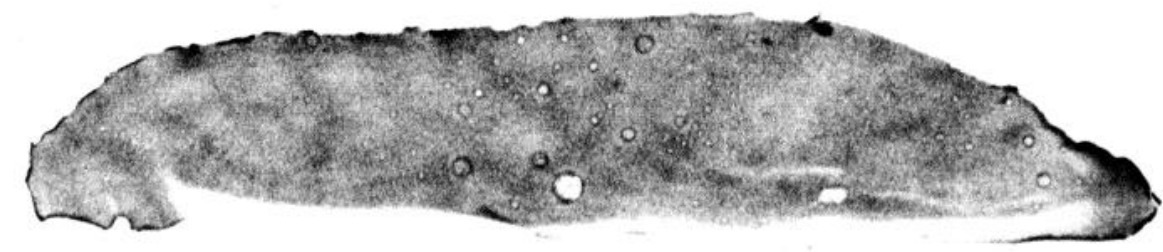

B

Figure 2. Patterns of DG uptake in 2 control cases. Both cases were shown achromatic, spatially diffuse stimuli. $A$, DG autoradiograph from ventral V2 from an animal that viewed a diffuse gray screen through both eyes during the period of DG uptake. The section is cut near-parallel to the layers, centered in layers 3B/4. Uptake within a given layer is very uniform; no trace can be seen of preferential uptake in any set of V2 stripes (as in Fig. 1). B, Another DG autoradiograph from a different animal, also taken from ventral V2, cut near-tangential and centered in layers 3B/ 4. The animal in $B$ was unanesthesized, unparalyzed, and kept in total darkness during the period of DG uptake. In this and one other animal treated identically, uptake is faintly higher in dark cytox stripes. This may indicate the presence of neural activity related to eye movements per se in at least one set of dark cytox stripes. Scale bar, $5 \mathrm{~mm}$.

type of DG uptake pattern: isolated columns confined largely to the interstripe areas (see Figs. 18 and 19, and below). The fact that V2 DG patterns were indistinguishable in the 2 cases further supports the idea that eye movements per se do not have a major effect on the types of DG patterns found in V2.

We next wondered about the pattern of DG uptake in response to a very generalizable, nonspecific visual stimulus. Would such a stimulus produce uniform uptake throughout V2, or would it produce higher uptake in one or more of the cytox stripe subdivisions? In order to test this question we showed 3 monkeys (cases $37,42,46$ ) a black-white square wave grating, binocularly, of systematically varied orientation (in $45^{\circ}$ steps), spatial frequency $(1,2$, and $4 \mathrm{c} / \mathrm{deg})$, velocity $\left(0.5^{\circ}-6 \% \mathrm{sec}\right)$, and direction (changed direction every $5 \mathrm{sec}$ ). In 2 of the 3 cases, this particular stimulus was used as a control case appearing in half of the visual field, and binocular disparity was left constant. In the remaining case, the stimulus covered the visual field on all sides to an eccentricity of $9^{\circ}$, and binocular disparity was varied systematically during the stimulus presentation over a range of $\pm 1^{\circ}$. [In examining results from all our cases, we have found that variations in binocular disparity (or lack thereof) have no obvious effect on the pattern of DG uptake in either V1 or V2 (see below).]

In animals viewing such generalized stimuli, DG labeling is not uniform; uptake in the thin and thick stripes is greater than that in the interstripes. This can be seen in Figure 3. In Figure $3 A$ the evidence is qualitative: stripes of higher DG uptake can be seen that are alternately thick and thin, and the distance between stripes of higher uptake is consistent with the average distance between adjacent stripes (as opposed to twice that dis- tance, as would be found between stripes of a single type).

In Figure 3, B, $C$, the DG evidence is linked to the cytox topography. The former is an autoradiograph taken from another animal that had been exposed to a visual stimulus identical to that used in Figure $3 A$; the latter is the same tissue section used in Figure $3 B$, after staining for cytox. In every dark cytox stripe (presumably thick plus thin), there is a corresponding increase in DG uptake in response to this very general visual stimulus.

There is also a little uptake in the cytox-light interstripe regions, especially in Figure $3 B$. In response to these extendedgrating stimuli, stimulus-related uptake is highest in the cytoxdark stripes but is not confined to it. Since single-unit studies have reported that interstripe cells are strongly end-stopped (Livingstone and Hubel, 1987), one would expect that stimuli without extended 1-dimensional edges, such as random-dot stimuli, would stimulate both stripe and interstripe areas more equally.

Figure 4 shows that result. This animal was shown (binocularly) a pattern of white, randomly positioned dots on a black background. The dots comprised $1 \%$ of the surface area, and they were drifted at $1 \% \mathrm{sec}$ across the visual field. The dots were drifted in only one direction because at the time we were doing the experiment for other reasons. The pattern of DG uptake in Figure 4 is less stripe-like than that in Figure 3, where extended gratings were used. The minor mottling that can be seen may be evidence of a directional architecture in V2, but the necessary control cases have not yet been done. At any rate, this case, in combination with cases like that in Figure 3, supports the idea that cells in the interstripes are end-stopped.

At one point in our analysis we needed accurate measurement 

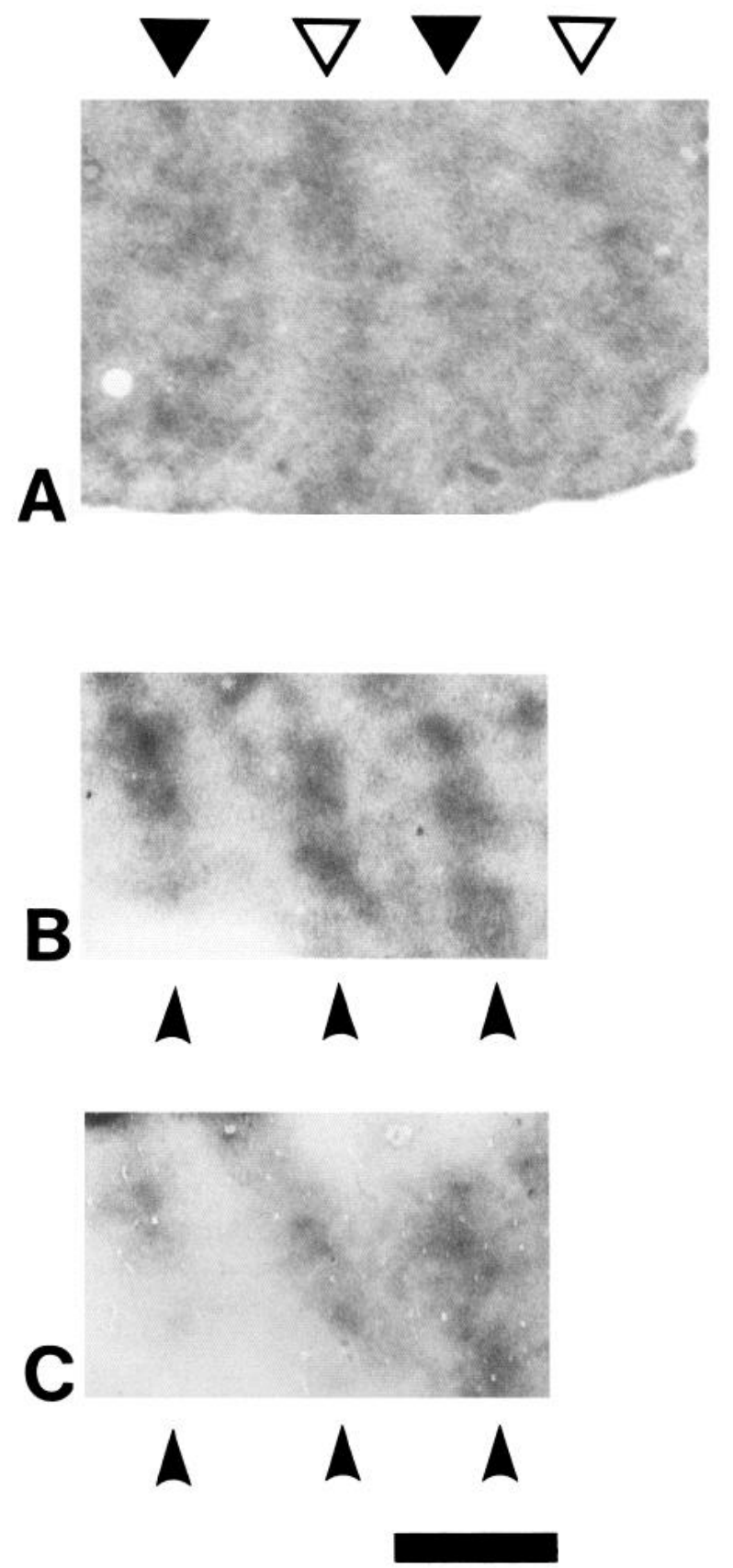

Figure 3. Very generalized grating stimulus produces high DG uptake in the thick and thin stripes. $A$ and $B$, Autoradiographs from sections cut near-tangential to the cortical layers of V2, centered on layers $3 B$ / 4. $C$, Section corresponding to the autoradiograph in $B$, after staining for cytox. $A$ is from one animal; $B$ and $C$ are from a different animal. Both animals weré shown, binocularly, an achromatic square wave grating of systematically varied orientation, spatial frequency, drift rate, and direction. In $A$, stripes of high uptake that alternate in thickness can be seen (filled triangle, thick; hollow triangle, thin). In other cases (e.g., panel $B$ ), a thick/thin distinction cannot be made with confidence, but every DG stripe has a counterpart in the cytox pattern (compare stripes above filled triangles in $B$ and $C$ ). Panel $B$ also shows some fain mottling in the interstripe regions. Scale bar, $2.5 \mathrm{~mm}$.

of the stripe geometry. We measured the width of the cytoxdark stripes, which were known to be "thin" and "thick" based on functional grounds (see DG results below), cytox interstripes, and the width of a full cycle of stripes. These data are presented in Table 2, for 3 species. Stripes judged to be "thin" on func- tional grounds (e.g., labeled by equiluminant color-varying stimuli, etc.) were nominally $0.7-0.8 \mathrm{~mm}$ wide, and "thick" stripes were closer to 1.1-1.2 mm wide. No species differences were found in the width of thin or thick stripes. Although the discrepancy is slight, a full cycle of stripes (a thin, a thick, plus 2 intervening interstripes) was slightly larger in the larger-brained $M$. arctoides $(4.25 \mathrm{~mm})$ than the small-brained $M$. assamensis $(4.07 \mathrm{~mm})$ and $M$. fasicularis $(3.96 \mathrm{~mm})$. This is due to the larger interstripe distance in $M$. arctoides.

We have been somewhat simplistic about whether DG uptake is high in a given set of cytox "stripes." In fact, neither the cytox nor the DG stripes are rectangular in shape. They are, instead, collections of small patches grouped together to form irregular stripes. Although the patches are irregular, they are often about $250 \mu \mathrm{m}$ in diameter and are spaced about $500 \mu \mathrm{m}$ apart (centerto-center).

The topography of the DG and cytox patches, which make up the stripes, is shown in Figure 5. Figure $5 A$ shows clear-cut DG patches from an animal (case 33) that was stimulated monocularly with spatially diffuse color variations. Because the visual stimulus used in Figure $5 A$ was so specific, it might be supposed that the patches occur only because we are labeling an organized subset of the total stripe topography. However, even in animals stimulated with very nonspecific visual stimuli, the DG stripes are made up of patches. Figure $5 B$ shows a DG autoradiograph taken from an animal (case 37 ) that was binocularly stimulated with a square-wave grating of systematically varied orientation, spatial frequency, velocity, disparity, etc. Again the DG stripes are clearly made up of patches.

Figure $5 C$ and $5 E$ are autoradiographs taken from an animal (case 20 ) that was stimulated with a low $(1 \mathrm{c} / \mathrm{deg})$ spatial frequency grating at systematically varied orientations, binocularly. Again, it can be seen that the V2 stripes are composed of tiny patches. Figure $5 C$ and $5 E$ are taken from sections $80 \mu \mathrm{m}$ apart. In these panels, the DG patch topography is essentially identical; thus, the patches are columnar, at least through layers $3 \mathrm{~B} / 4$.

In this animal the cytox stripes also showed up nicely. Figure $5 D$ and $5 F$ show the sections used to produce Figure $5 C$ and $5 E$, after staining for cytox. The interesting thing about this comparison is that both the DG and the cytox staining show V2 stripes, but the correspondence is not perfect. The DG extends further than the limits of the cytox stripes, and it is somewhat different in shape and emphasis even within the cytox limits. We do not yet know why this is so.

\section{Retinotopic organization}

As in V1, the receptive fields in V2 are arranged so that they reflect the initial geometry of the photoreceptors, in addition to subsequent cortical transformations. Based on electrophysiological maps, the major transformations in the retinotopy of $\mathrm{V} 2$ (relative to striate cortex) include (1) an enlargement of the average receptive field by a factor of about 3 (Baizer et al., 1977; Van Essen and Zeki, 1978; Gattass et al., 1981; Foster et al., 1985), with corresponding increases in receptive field scatter; (2) a decrease in overall area by a factor of about $20 \%$ (Gattass et al., 1981); (3) a division of the overall map into 2 halves, separated along the horizontal meridian (Allman and Kaas, 1974).

There are also a number of unanswered questions about the $\mathrm{V} 2$ retinotopy. For instance, there are actually 2 complete maps of the visual field feeding into striate cortex (one from each eye), 

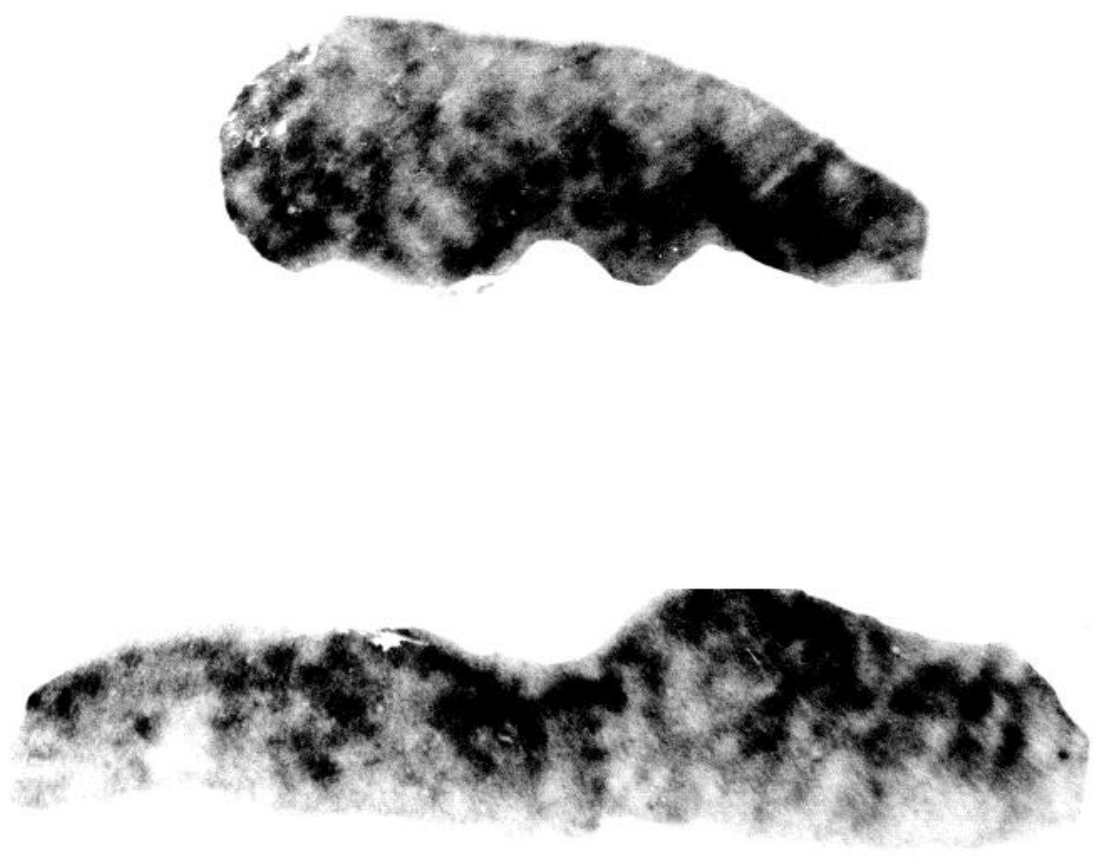

Figure 4. Stimuli without extended contours produce high DG uptake in both stripes and interstripes. In this case, the monkey saw a pattern of random dots (white on black, $2 \%$ of surface area), drifting at $1 \% \mathrm{sec}$ horizontally, through both eyes. There is some mottling of the pattern, which may or may not indicate a directional architecture. The point we wish to emphasize is that the random dots produced high uptake in the interstripes, although extended gratings do not (see Fig. 3). This is consistent with electrophysiological reports that cells in the interstripes are end-stopped; such cells should respond well to low-density random dot patterns such as these, but not to extended gratings. Scale bar, $5 \mathrm{~mm}$.

and with DG these 2 maps can be traced all the way through striate cortex into the layers that project to V2 (Tootell et al., 1988 b). Thus, are there 1 or 2 retinotopic maps in area V2, particularly in input layer 4 of V2?

In order to test some of these ideas we showed a number of animals visual stimuli that included discrete visuotopic borders, and we used DG to label the discreteness of the corresponding retinotopic border representations in V2. In this section we report on data from 3 of these cases.

Data from 2 of these cases (cases 9, 13) are shown in Figure 6 , from both striate cortex (panels $A, C$ ) and from retinotopically corresponding regions of $\mathrm{V} 2$ from the same hemisphere (panels $B$ and $D$, respectively). Figure $6 A$ and $6 C$ illustrate the DG maps in striate layers $2+3$, which project to layer 4 of $\mathrm{V} 2$ (panels $B$ and $D$ ). In all panels, the visual stimulus consisted of a line of small, rectangular black-and-white checks that were counterphased on a stable gray background (stimulus illustrated in Tootell et al., 1982, 1988b). The width of each check was $0.13^{\circ}$, and the length of each check was randomized about a mean of $0.27^{\circ}$ (range $\left.=0.18^{\circ}-0.4^{\circ}\right)$. Figure 6 includes the representation from about $2^{\circ}-4^{\circ}$ eccentricity, along a ray running $45^{\circ}$ to the vertical or horizontal meridia. In panels $A$ and $B$, the stimulation is

Table 2. Measurements of cytox subdivisions in several species of macaque

\begin{tabular}{llllll} 
Species & \multicolumn{2}{l}{ Thick stripe } & Thin stripe & Interstripe & Full cycle \\
\hline M. arctoides & $x=$ & $1.16 \mathrm{~mm}$ & $0.74 \mathrm{~mm}$ & $1.27 \mathrm{~mm}$ & $4.25 \mathrm{~mm}$ \\
& $o=$ & 0.10 & 0.08 & 0.19 & 0.52 \\
& $N=$ & 8 & $N=6$ & $N=8$ & $N=6$ \\
M. assamensis & $x=$ & $1.16 \mathrm{~mm}$ & $0.73 \mathrm{~mm}$ & $1.14 \mathrm{~mm}$ & $4.07 \mathrm{~mm}$ \\
& $o=$ & 0.10 & 0.08 & 0.18 & 0.39 \\
M. fasicularis & $N=$ & 8 & $N=7$ & $N=9$ & $N=7$ \\
& $x=$ & $1.11 \mathrm{~mm}$ & $0.74 \mathrm{~mm}$ & $1.07 \mathrm{~mm}$ & $3.96 \mathrm{~mm}$ \\
& $o=$ & 0.07 & 0.09 & 0.18 & 0.43 \\
& $N=$ & 10 & $N=9$ & $N=11$ & $N=11$
\end{tabular}

The cytox-dark regions were defined as either "thick" or "thin" on the basis of DG labeling in those cases where this was unambiguous (see text for details). No species differences in stripe width were apparent. The width of a full cycle of stripes (a thick, a thin, and 2 intervening interstripes) was slightly wider in the larger-brained ( $M$. arctoides) than in the smaller-brained macaques ( $M$. fascicularis and $M$. assamensis). Measurements were made in layers $3 \mathrm{~B} / 4$ at or near the $\mathrm{V} 1 / \mathrm{V} 2$ border. 
Figure 5. Patchiness in the topography of the V2 stripes. $A, B, C$, and $E$, DG autoradiographs from sections cut near-parallel with the layers, passing mostly through layers $3 B / 4$. Quite different visual stimuli were used to produce the different DG patterns. $A$, From an animal that monocularly viewed spatially diffuse variations in color. $B$, From an animal that viewed (binocularly) a black-white square-wave grating of systematically varied orientation, direction, drift rate, and spatial frequency. $C$ and $E$, Autoradiographs from adjacent sections of a single animal that was shown an achromatic, low spatial frequency, sinusoidal grating at varied orientations, drift rates and directions. $D$ and $F$, Sections corresponding to $C$ and $E$, after staining for cytox. $C-F$ show good views of a thin (right) and thick (left) stripes. From evidence detailed elsewhere, we know that the stripe in $A$ is a thin stripe. The pattern of high uptake in $B$ could be in a thick or thin stripe, but it appears thin. In both the DG and cytox stripe patterns, it can be seen that the V2 "stripes" are actually made up of patches. The topography of the patches in adjacent sections is very similar (cf. $C$ and $E$ ). Therefore, the patches are relatively columnar in shape, at least through layers $3 \mathrm{~B} / 4$, where the patches are most clearly defined. Comparison of the DG and cytox patches in the same section (e.g., $C$ vs $D, E$ vs $F$ ) shows some significant differences, at least when produced by some visual stimuli. Scale bar, $2.5 \mathrm{~mm}$.
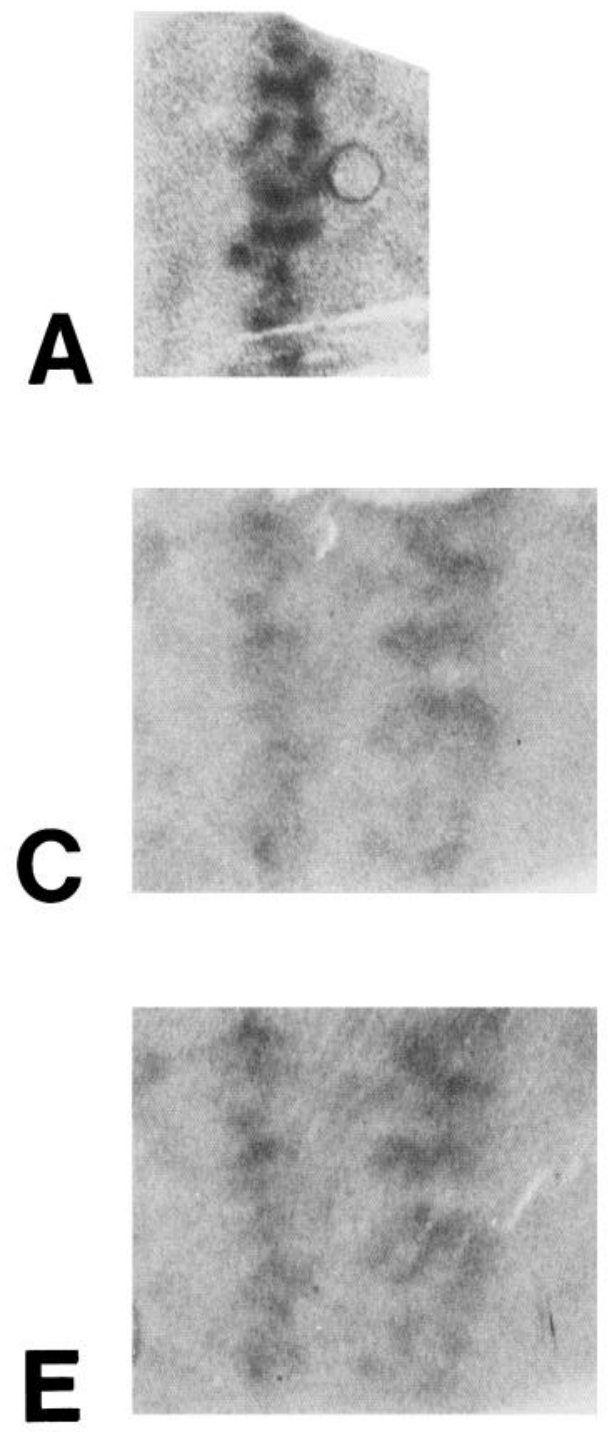
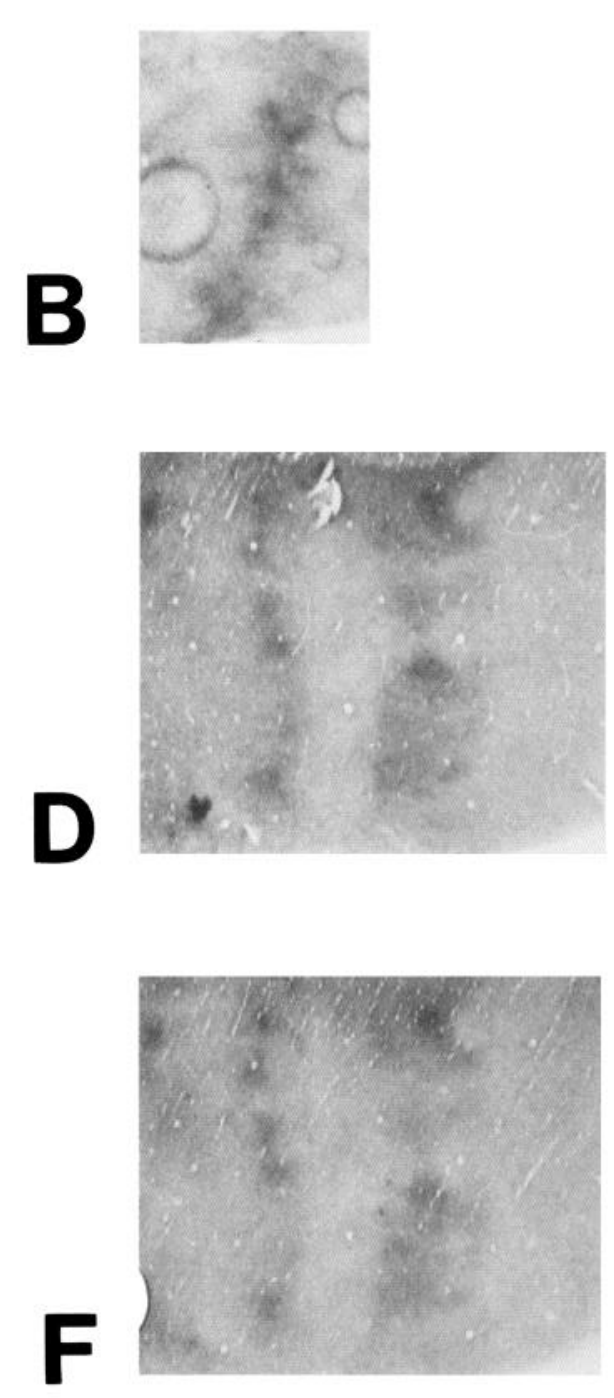

monocular; in panels $C$ and $D$, the stimulation was binocular but slightly out of alignment in places, due to a minor cyclorotation of the eyes.

Several points are salient. First of all, there is very little decrease in the resolution of the DG maps between the output layers of V1 (Fig. 6, $A, C$ ) and the input layers of V2 (Fig. 6, $B$, $D$ ). This was initially surprising to us because the average receptive field size in V2 has been described as 3 times larger than that in V1, and presumably, receptive fields 3 times larger would produce correspondingly blurrier DG maps. However, receptive fields in striate layers $2+3$ may be larger than those in the striate input layer 4Cb (Hubel and Wiesel, 1974; Tootell et al., $1988 \mathrm{~b}$ ), and from the DG evidence we presume that the receptive fields in layers $3 \mathrm{~B} / 4$ of $\mathrm{V} 2$ are smaller than those in extragranular layers of V2 (see below). Thus, we are presumably looking at the V1-V2 link in a longer chain of increasingly larger receptive fields, all the way from the input layers of V1 through the output layers of V2. So it is less surprising to find just a small increase in retinotopic spread between the V1 output lay- ers and the V2 input layers. Quantitatively, measurements of the fall-off of DG uptake in the layers 3B/4 maps of V2 (Fig. $6, B, D$ ) are about $400-700 \mu \mathrm{m}$ in extent (half-amplitude at halfwidth; measured as in Tootell et al., 1988b). DG fall-offs in striate layers $2+3$, measured comparably, are about $250-500$ $\mu \mathrm{m}$ in extent. The measurements in V2 should be taken with a grain of salt, however, because the DG patterns are relatively faint (see Discussion).

A second interesting feature of the $\mathrm{V} 2$ retinotopy is that there is an apparent lack of ocular dominance architecture in the V2 maps (Fig. 6, $B, D$ ), although there is an unambiguous segregation of the 2 maps into corresponding sets of ocular dominance stripes in the $\mathrm{V} 1$ maps (Fig. 6, $A, C$ ). This corroborates other DG evidence against an ocular dominance segregation in V2 (see below). Thus, the DG evidence indicates that the 2 input maps that arrive in striate layer 4 remain segregated throughout the striate cortex and are architecturally integrated in the input layers of the second-tier striate-recipient area V2.

A third point about the retinotopic pattern in Figure 6 is that 

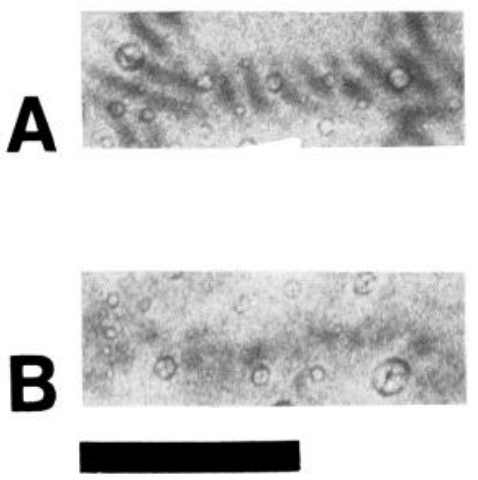
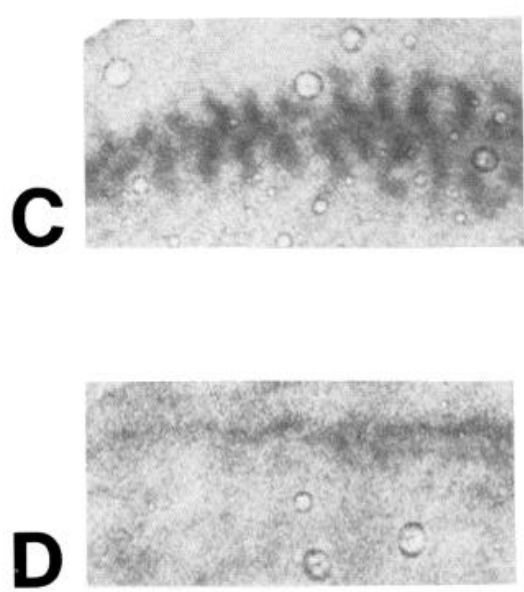

Figure 6. Retinotopic resolution of V2, in comparison to V1, when using small visual stimuli. All panels show DG autoradiographs from sections cut parallel or near-parallel with the cortical layers. $A$ and $C$ are taken from area $\mathrm{V} 1 ; B$ and $D$, from the corresponding visual field representation in area V2. The stimulus in all cases was a row of small, blinking black-and-white checks on a stable field of homogenous gray. In $A$ and $B$ the stimulus was viewed monocularly; in $C$ and $D$ (taken from a different animal), a very similar stimulus was viewed binocularly, but the image was slightly cyclorotated in one eye relative to the other. The 2 monocular images are converged in cortex only at the far left edge of $C$ and $D$, and the images become progressively diverged towards the right of the figure. The cortical representation of the row of black-and-white checks appears as a row of ocular dominance stripes in striate cortex, either because only one eye was stimulated $(A)$ or because the 2 monocular images were unconverged on the cortex $(C)$. No such ocular dominance substructure appears in the representation of the same stimulus in area V2. In $B$ and the left half of $D$, there is a single continuous representation of the stimulus ring. In the right half of $D$, there are 2 parallel stripes, presumably representing the 2 unconverged monocular images. $A$ and $C$ are taken from layers $2+3$ of $\mathrm{V} 1$, which project to layer 4 of V2 $(B$ and $D)$. The retinotopic resolution of the map in layer 4 of $\mathrm{V} 2$ is degraded very little relative to that in V1. Scale bar, $5 \mathrm{~mm}$.

the overall width of the retinotopic pattern is distinctly less in $\mathrm{V} 2$ than in corresponding regions of V1. Some of this may be apparent rather than real, due to a consolidation of information from 2 sets of ocular dominance stripes (in the V1 pattern; Fig. $6, A, C$ ) into a single unified pattern in the V2 map (Fig. $6, B$, $D$ ). However, some of the reduction may be due to the smaller overall size of V2, relative to V1 (Gattass et al., 1981), or to possible nonlinearities in DG uptake (the "tip of the iceberg" problem).

A final observation on the retinotopic organization of $\mathrm{V} 2$ is illustrated in another case (case 26) in which we presented (monocularly) a very general stimulus (a black-white square wave grating of 1,2 , and $4 \mathrm{c} / \mathrm{deg}$, presented at varied orientations in $45^{\circ}$ steps, at varied velocities and directions) which was confined to a narrow stripe $\left(1.4^{\circ}\right.$, or $0.7^{\circ}$ on each side of the midline) centered along the vertical meridian, bordered by a uniform gray. In V2, this stimulus produced a stripe-shaped pattern of high DG uptake with a clear retinotopic border between the representation of the grating and the uniform gray background (see Fig. 7). In V1, clear ocular dominance stripes appeared (see fig. 8 of Tootell et al., 1988b), but in V2 the only variation in DG uptake within the grating representation was a modulation of uptake between cytox stripe and interstripe areas in all layers of V2. The modulation of uptake between stripe and interstripe areas is consistent with tests of V2 uptake to a very general visual stimulus viewed binocularly (see Fig. 3); the lack of additional topographic variation is further evidence against the existence of ocular dominance columns in V2.

In $\mathrm{V} 1$, it proved possible to exploit the highly organized retinotopic representation to do split-field experiments (e.g., Tootell et al., 1988a-e). In those experiments, the DG effects of one stimulus could be compared with the DG effects of its appropriate control stimulus within the same hemisphere by positioning the 2 stimuli on opposite sides of the horizontal merid- ian. In Figures 6 and 7, it is obvious that the retinoptic organization of area V2 is discrete enough so that we could do analogous split-field comparisons of different visual stimuli in V2. A number of such stimulus comparisons are presented below.

\section{Ocular dominance}

Above, we presented data that suggested a lack of ocular dominance segregation in V2. In this section we present more direct evidence on this point.

In one animal (case 50 ) we presented a very general stimulus (a black-white square wave grating of 1,2 , or $4 \mathrm{c} / \mathrm{deg}$, presented at varied orientations in $45^{\circ}$ steps, at various velocities and directions). By carefully positioning a diffuse gray occluder along the horizontal meridian in front of one eye, we were able to arrange it so that the stimulus was viewed binocularly in the lower half of the visual field and monocularly in the upper half. The monocular-binocular split was confirmed as lying along the horizontal meridian by examining the DG topography in V1: ocular dominance stripes appeared in ventral striate cortex, but they did not occur within dorsal striate cortex (see fig. 9 of Tootell et al., 1988a).

Patterns of DG uptake from corresponding (that is, monocularly vs binocularly stimulated) regions of V2 are shown in Figure 8. As in analogous cases shown earlier (Figs. 3, 7), DG uptake is higher in stripe-like regions corresponding to the dark cytox stripes, and there is no obvious difference in the pattern of uptake between monocularly and binocularly stimulated regions. Figure 8 is confined mostly to striate-recipient layers $3 \mathrm{~B} /$ 4 , but differences between stimulation conditions did not appear in other layers either. Towards the right of the figure, it appears that one (thick) stripe is not labeled; this may be due to an overall lighter labeling of thick stripes plus variability or to the fact that the stimulus border was represented at this region. 

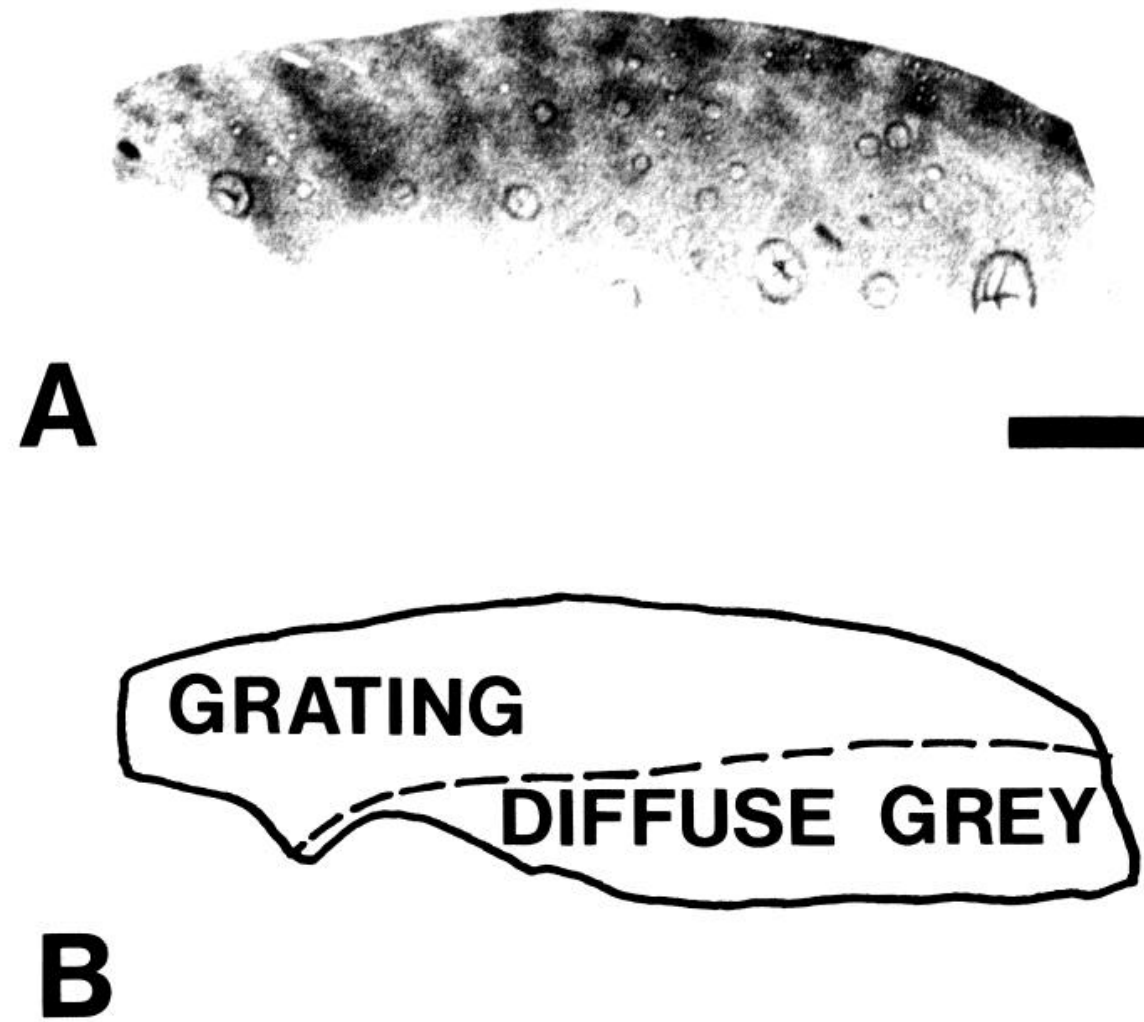

Figure 7. Retinotopic resolution of $\mathrm{V} 2$, using a larger stimulus. $A$ is from a section cut near-parallel to the cortical layers, centered on layer 4 , and it includes regions of dorsal V2 ranging from a representation of the fovea (far left) to about $8^{\circ}$ (far right). The animal in this case was shown, monocularly, a square-wave grating of varied orientation, direction, spatial frequency, and drift rate. The grating was confined to a thin stripe (1.4 $4^{\circ}$ total width, $0.7^{\circ} /$ hemisphere), centered on the vertical meridian, and it was surrounded by a field of uniform gray. The limit of the representation of the grating versus surround in $A$ is diagrammed in $B$. The retinotopic limits produced by such a large stimulus appear slightly less sharp than those produced by smaller stimuli in Figure 6 . However, the retinotopic resolution is clearly good enough to do "split-field" comparisons of the effects of different visual stimuli in correspondingly different parts of the visual field. Stimulus-related uptake within the V2 stripes appears to extend further in the interstripe regions, giving the border a scalloped appearance. It is not clear whether this retinotopic "scalloping" is real or is due to a lower overall uptake in the interstripe regions in response to this stimulus (e.g., Fig. 3). Scale bar, $5 \mathrm{~mm}$.

\section{Orientation}

Many cells in area V2 are orientation-selective, responding well to some orientations but not to others (Hubel and Wiesel, 1970; Baizer et al., 1977; Zeki, 1978; Hubel and Livingstone, 1987). From tangential electrode penetrations, it appears that cells of like orientation are grouped together in V2 (Hubel and Wiesel, 1970; DeYoe and Van Essen, 1985; Shipp and Zeki, 1985; Hubel and Livingstone, 1987) as they are in area V1 (Hubel and Wiesel, $1962,1968)$. There is even a single report of DG periodicities that may represent DG orientation columns in V2 (Livingstone and Hubel, 1982), although this report does not include an allorientation control case. In this section we examined V2 for DG evidence for an orientation-specific architecture.

Recall that in Figures 3, 7, and 8, a very general visual stimulus (varied in orientation, among other parameters) produces a pattern of DG uptake that is highest in both sets of dark cytox stripes and lower in the interstripes. As an initial test of orientation specificity, we presented an animal (case 8) with a stimulus that was identical to those used above, except that orientation was held constant instead of varied. The stimulus was thus a black-white square-wave grating of varied spatial frequency $(1,2$, or $4 \mathrm{c} / \mathrm{deg})$, drifting in both directions at varied velocities at a horizontal orientation. The grating was presented binocularly, and the binocular disparity was not varied.
Autoradiographs from V2 of this case are shown in Figure 9. Clear periodicities are present which are about $300-500 \mu \mathrm{m}$ in diameter, separated by about $400-1000 \mu \mathrm{m}$, arranged in a semiregular array. The position of the periodicities in the array remains essentially constant from cortical surface to white matter, so the periodicities are columnar in shape. Since such periodicities are absent in all-orientation cases, the periodicities presumably represent orientation columns in V2. In some places (top section of Fig. 9), the topography of the presumptive orientation columns is elongated on one side, as one would expect from tangential single-unit penetrations showing orientationspecific cells tuned to the same orientation for long distances through V2 (Hubel and Livingstone, 1987). In other regions, the orientation columns are not elongated. Superimposed on the dark columns, one can also see moderate levels of uptake in stripes running vertically through Figure 9. Based on the comparisons to the cytox topography in other animals (Tootell et al., 1988f), we presume these to be the thin stripes.

As further evidence that these DG columns represent orientation-specific regions of V2, we presented a grating of one orientation (in this case, vertical) and a grating of varied orientations (in $45^{\circ}$ steps) in adjacent halves in a split-field experiment (case 22). Again, the square-wave grating was varied in spatial frequency $(1,2$, and $4 \mathrm{c} / \mathrm{deg})$, moved in both directions at a wide range of velocities, and presented monocularly. 

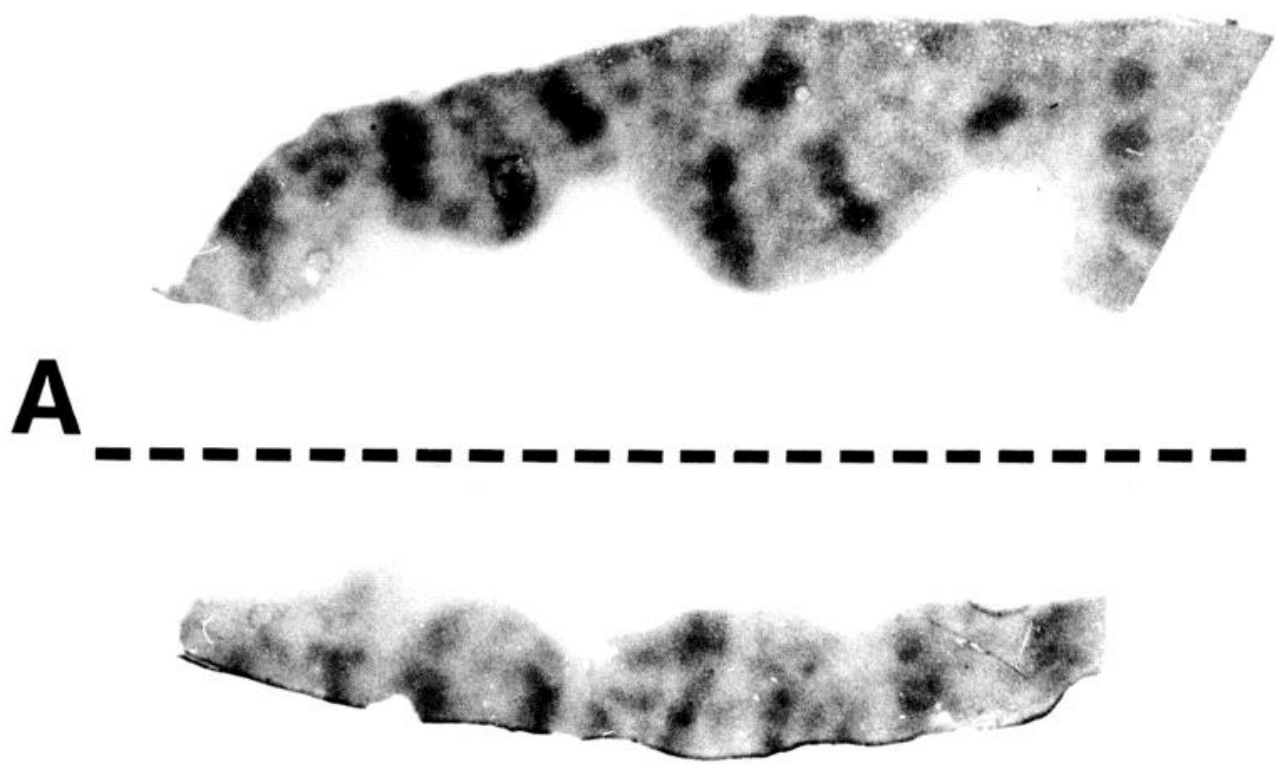

B

Figure 8. Split-field comparison of the effects of monocular and binocular visual stimulation. $A$ and $B$ are autoradiographs from the same single tissue section, encompassing the representation of the inferior visual field $(A)$ and the superior visual field $(B)$. This animal was stimulated monocularly in the superior visual field $(A)$ and binocularly in the inferior visual field $(B)$. The stimulus was a very general one: a square-wave grating of systematically varied spatial frequency, orientation, direction, and drift rate. In the binocular regions, the pattern of uptake is identical to that described earlier in response to the same visual stimulus (e.g., Fig. 3): uptake is high in the dark cytox stripes. In some portions of $A$, the pattern looks artifactually spotty due to the plane of section; in $B$, the pattern of DG uptake is qualitatively indistinguishable from that in $A$. In this and other tests, there is no DG evidence for ocular dominance columns in V2. Scale bar, $5 \mathrm{~mm}$.

Results from a single section through V2 are shown in Figure 10. As expected, isolated columns of orientation-specific uptake appear in the portion of $\mathrm{V} 2$ stimulated with a single-orientation stimulus (Fig. 10A) but not in the portion of V2 stimulated with a grating of varied orientations (Fig. 10B).

A very similar case, tested binocularly, supports this conclusion. In this case, we used a split-field stimulus to compare the effects of one orientation with that of 2 orientations. In area V1, stimuli of one orientation produce clear DG periodicities, but stimuli of 2 orthogonal orientations produce patterns of uptake that are close to uniform (R. B. H. Tootell, S. L. Hamilton, M. S. Silverman, and E. Switkes, unpublished observations). In both halves of the stimulus, a drifting square-wave grating of varied spatial frequency, velocity, and direction appeared. In the bottom half, the grating was horizontal; in the top half, the grating was alternately horizontal and vertical. The stimulus was viewed binocularly.

The result of this stimulus is shown in Figure 11. Again, panels $A$ and $B$ represent portions of $\mathrm{V} 2$ from a single tissue section, showing the dorsal and ventral portions of V2, respectively. In dorsal V2 (Fig. 11 A), the single-orientation columns are obvious and typical of those described earlier. In ventral V2 (Fig. 11B), the DG pattern is more uniform, but some faint periodic mottling occurs. Our interpretation here is that the faint periodicities represent the sum of the horizontal and vertical orientation columns in V2. The 2-orientation periodicities are presumably fainter than the 1-orientation periodicities because in the 2-orientation condition, cells were being stimulated with a preferred orientation only half the time. This interpretation is con-

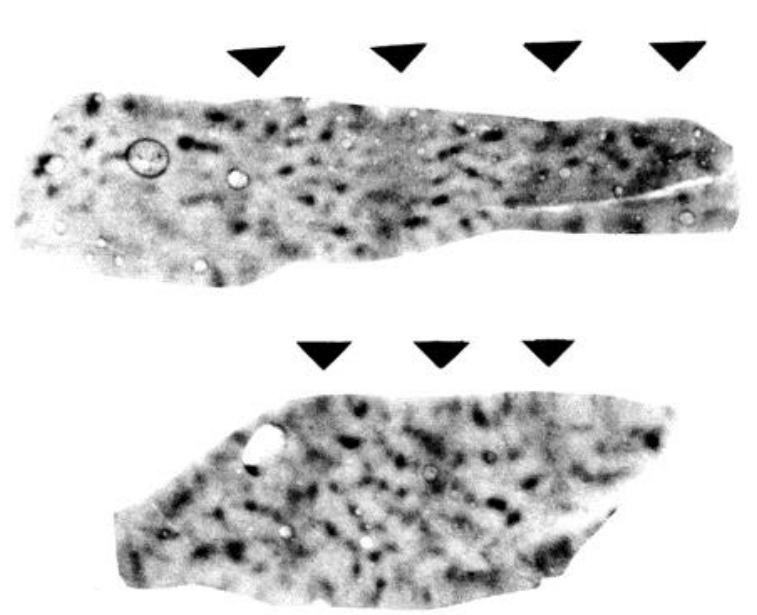

Figure 9. Orientation columns in V2. The DG autoradiographs here are from sections cut near-parallel to the cortical layers, including dorsal and ventral portions of central V2. The stimulus was a horizontal squarewave grating of varied spatial frequency, direction, and drift rate. The grating was viewed binocularly. The most prominent feature of this autoradiograph is the dark spotty regions of high uptake which represent orientation columns (see controls below). Fainter, blurry stripes of moderate uptake (filled triangles) can also be seen particularly well in the lower of the 2 sections, running near-vertical through the figure. Other evidence (Tootell et al., 1988f) indicates that these correspond to the thin cytox stripes. Scale bar, $5 \mathrm{~mm}$. 

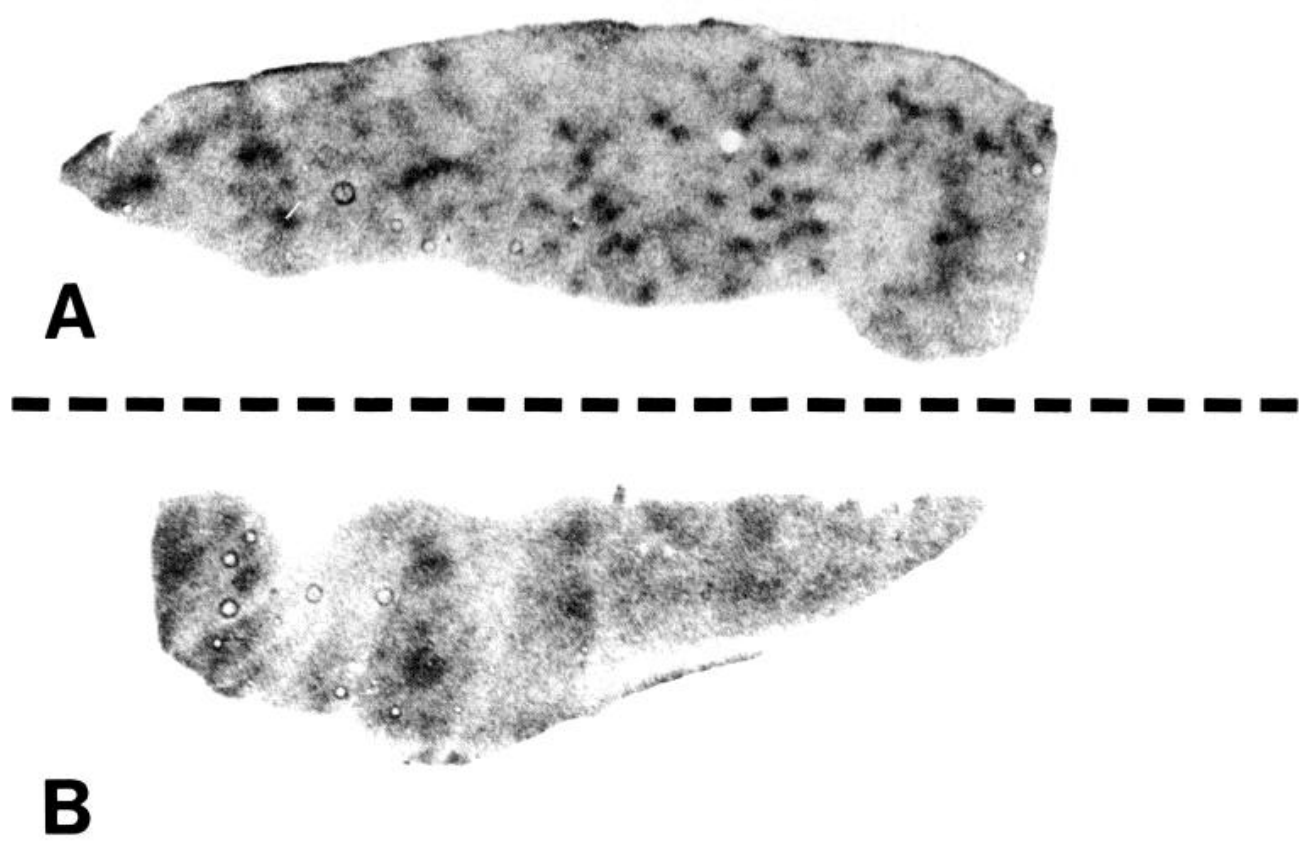

Figure 10. Split-field evidence for orientation columns. $A$ and $B$ are taken from the same tissue section, including the central representation of the inferior visual field $(A)$ and the superior visual field $(B)$. The stimulus in both halves of the visual field was a grating of systematically varied spatial frequency, direction, and drift rate. In the inferior visual field $(A)$, the grating was presented at a single orientation. In the superior field $(B)$, the grating was presented at systematically varied orientations. The stimulus was viewed monocularly. In $A$, spotty regions of higher uptake can be seen which are not found in $B$. This is within-animal evidence for orientation columns in V2. Scale bar, $5 \mathrm{~mm}$.

sistent with results from double-labeling of DG orientation columns in V2 (Tootell et al., 1988f).

As in macaque $\mathrm{V} 1$, the distribution of $\mathrm{V} 2$ columns for a given orientation does not seem to be biased within the visual field.
That is, columns of cells tuned to vertical do not cluster near the representation of the vertical meridian and away from the horizontal, and likewise for other possible permutations (see Figs. 9-11).
Figure 11. Split-field comparison of the effects of a grating at one orientation versus 2 orthogonal orientations. As in Figures 3 and 10, the autoradiographs in $A$ and $B$ are taken from a single section, from representations below and above the horizontal meridian ( $A$ and $B$, respectively). The fovea is represented at the far right of each panel, and more peripheral regions to the left. The stimulus was a grating of systematically varied spatial frequency, drift rate, and direction. In $A$, the grating was presented at a horizontal orientation. In $B$, the grating was presented at an orientation that was alternately vertical or horizontal. The grating was viewed binocularly. In $A$, there are spotty regions of high uptake which we take to be horizontal orientation columns. In $B$, labeling is much more uniform (due to the decrease in orientation specificity), but very faint periodicities can be seen, particularly in the center-right of the section. As far as we can tell, these faint periodicities appear to have approximately twice the packing density of those in $A$. We take them to be the sum pattern of the horizontal plus vertical orientation columns. Scale bar, $5 \mathrm{~mm}$.
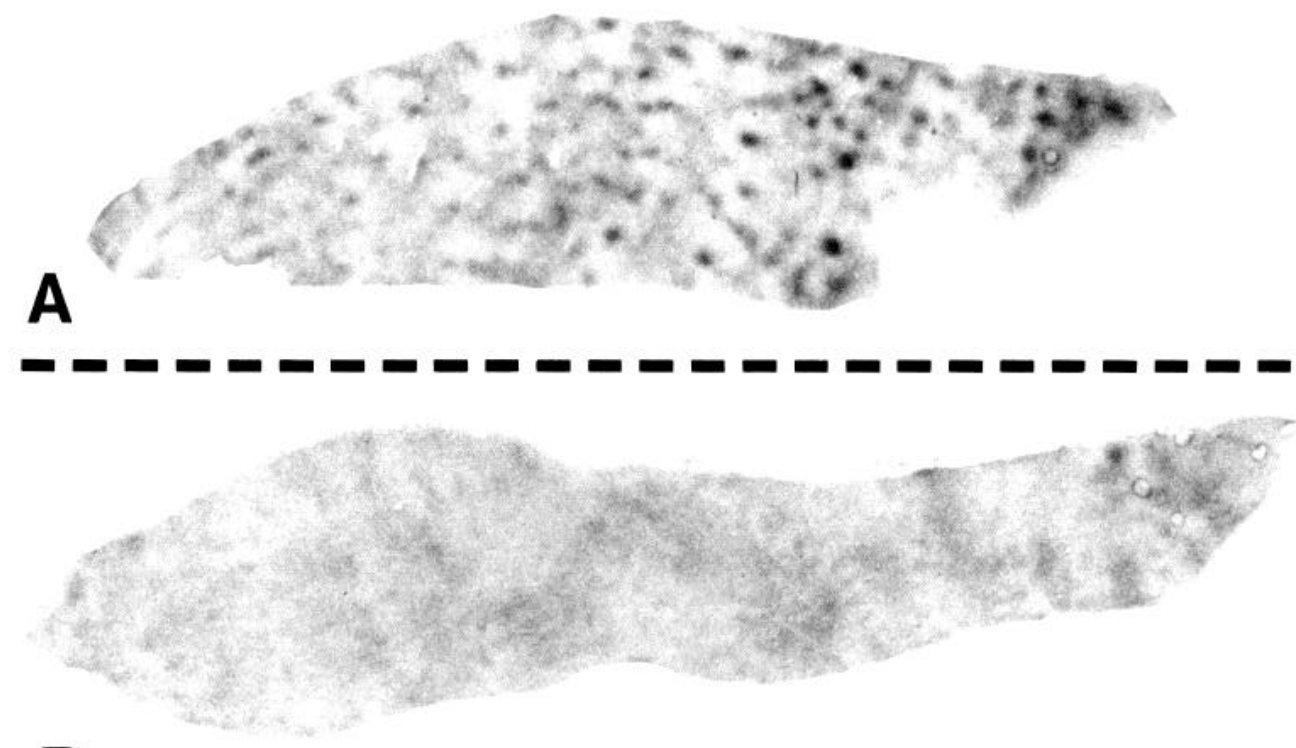

B 

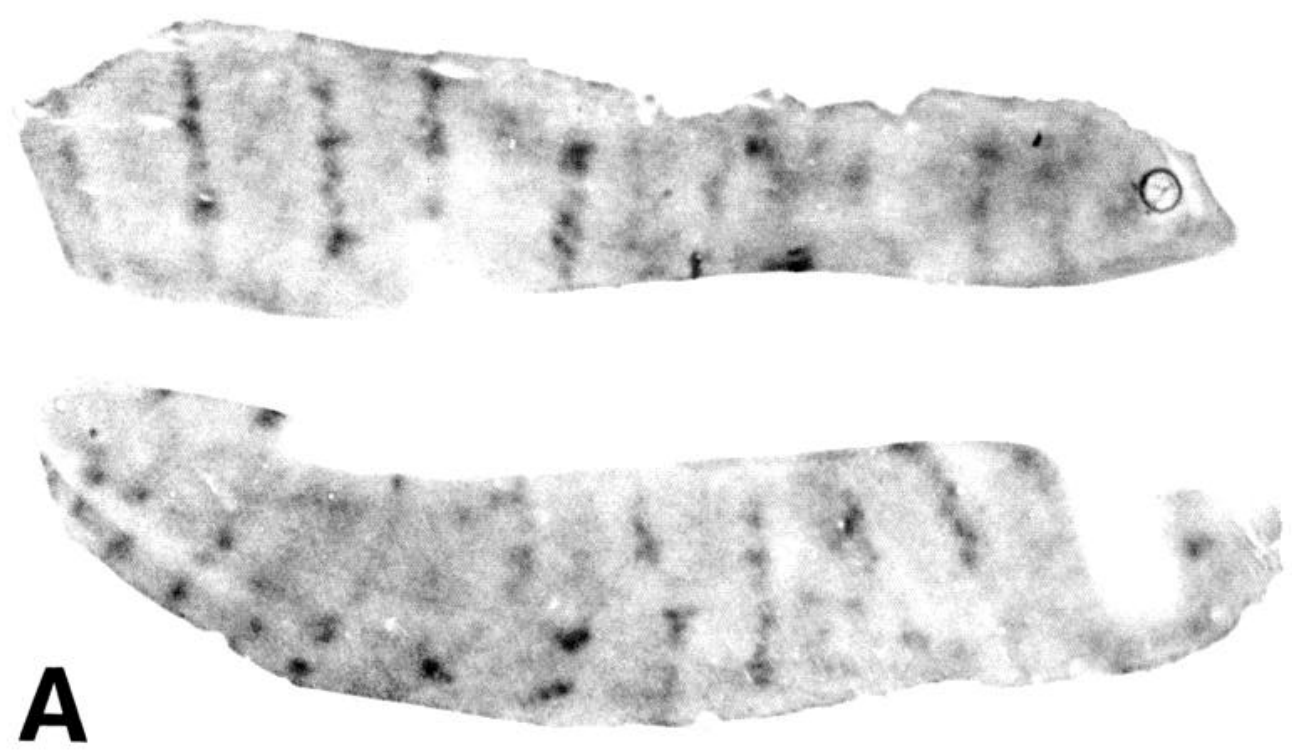

Figure 12. Typical result of stimulation with spatially diffuse variations in color $(A)$ versus spatially diffuse variations in luminance $(B) . A$ and $B$ are autoradiographs from sections cut neartangential to the cortical layers, centered on layer 4, from the central representation of V2 in one hemisphere. The animal in $A$ viewed spatially diffuse equiluminant red-gray variations, binocularly. The animal in $B$ viewed a stimulus that was spatially and temporally identical, in which the variation was along the black-white axis. Such spatially diffuse variations produce high uptake in one set of V2 stripes when modulated in color, but not when modulated in luminance. The stripes of high uptake in $A$ are about $4 \mathrm{~mm}$ apart, which is consistent with being in every other dark cytox stripe. Scale bar, $5 \mathrm{~mm}$.
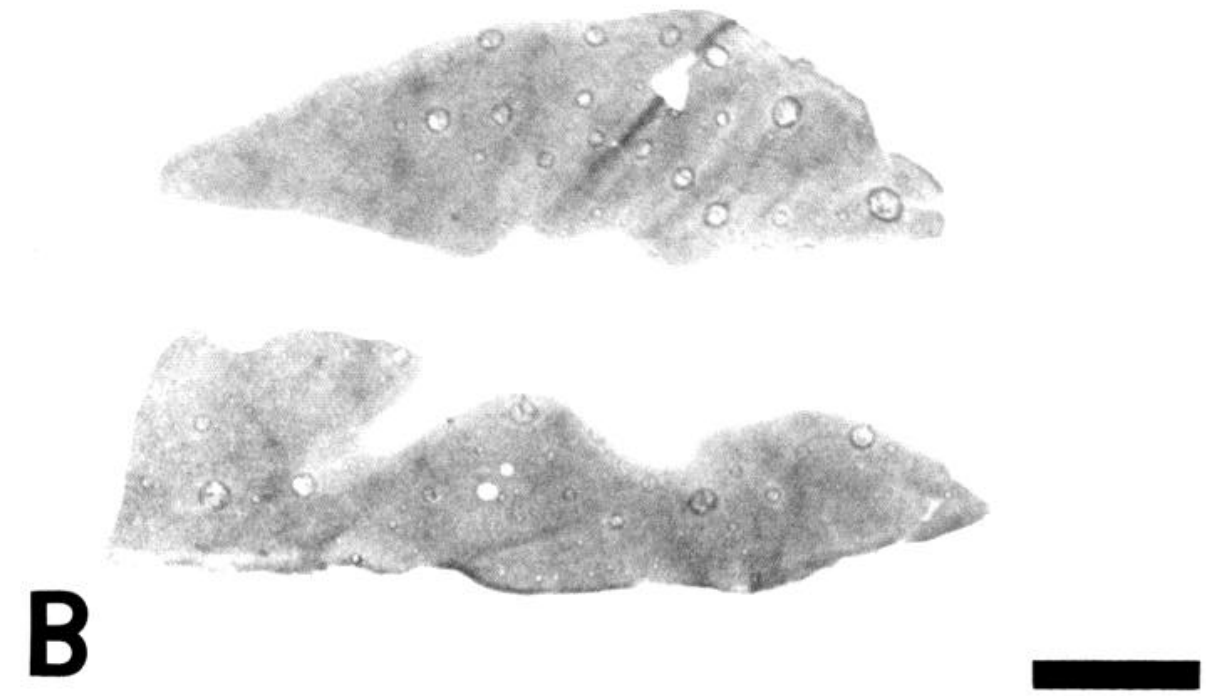

\section{Color}

In DG studies of striate cortex, flickering, spatially diffuse color stimuli produce high uptake in the cytox blobs, whereas spatially diffuse black-white stimuli produce little or no uptake anywhere in striate cortex (Tootell et al., 1988c). These DG results correlate nicely with the behavior of single cells in striate cortex generally (e.g., Thorell, 1980; Thorell et al., 1984) and in the upper-layer blobs and interblobs specifically (Livingstone and Hubel, 1984). The robust DG uptake in the blobs in response to spatially diffuse color stimuli is presumably mediated by the nonoriented single-opponent (and 1 1/2-opponent) color cells that comprise a significant percentage of all blob cells (Livingstone and Hubel, 1984).

Since the blobs project to the thin stripes in V2 (Livingstone and Hubel, 1983), one might expect that visual stimuli which activate the blobs would produce strong activation of the thin stripes as well. Spatially diffuse achromatic variations in luminance, which produce very little activation of striate cortex, should presumably produce very little activation of $\mathrm{V} 2$ as well, if we disregard the contribution of other inputs to V2.

These expectations were met very nicely in the DG data from V2. In 2 representative cases (cases 6,7 ), each animal viewed a color monitor binocularly. The whole monitor screen was programmed to change either from equiluminant red-to-gray (Fig. $12 A$ ) or from black-to-white (Fig. $12 B$ ), at $0.5-2 \mathrm{~Hz}$ in a temporal square wave. In V2, spatially diffuse variations in color produce high uptake in one set of stripes, and spatially diffuse variations in luminance produce very little uptake at all (see Fig. 12). In the color-varying cases (e.g., Fig. 12A), the spacing between stripes of high uptake is about $4 \mathrm{~mm}$. This is a distance that almost certainly indicates uptake in either the thin or thick cytox stripes, but not in both.

These measurements are bolstered by more compelling data. 

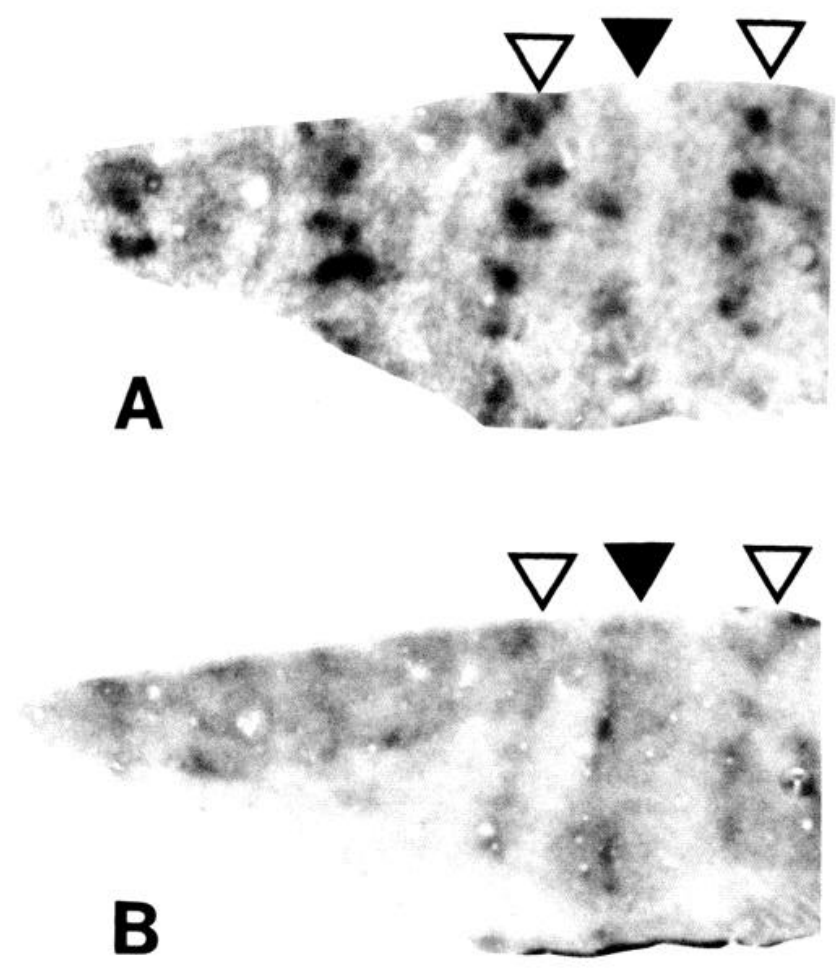

Figure 13. Spatially diffuse variations in color produce high uptake in every other dark cytox stripe. $A$, Pattern of DG uptake in response to binocular viewing of spatially diffuse variations in color. $B$, Cytox stain in the same section. In this animal, dark cytox stripes were visible in V2. In places they could be described as thick or thin (filled or unfilled triangles, respectively) based on the width of the cytox-stained stripe in this and adjacent sections. In response to this stimulus, the patchy stripes of high DG uptake appear to lie in the thin but not the thick stripes. Scale bar, $5 \mathrm{~mm}$.

In some cases, cytox histology revealed dark stripes in V2. In these cases, it was possible to confirm that the stripe-like regions of high uptake in response to spatially diffuse color stimulation lie on every other set of dark V2 stripes (see Fig. 13). Presumably these are the thin stripes because the DG uptake patterns are quite thin (see Figs. 12,13) and because this follows logically from the HRP results of Livingstone and Hubel (1983).

There is also DG evidence for color-opponent cells with strong suppressive surrounds (e.g., double-opponent) in V2. In the V1 blobs, such cells have been shown using electrophysiological mapping methods (Livingstone and Hubel, 1984). In area V1, we were able to produce supportive DG evidence for such double-opponent cells by showing that sinusoidal color-varying gratings of low-to-medium spatial frequency produced more uptake than spatially diffuse color variations when using identical equiluminant hue pairs (e.g., fig. 16 of Tootell et al., 1988c, case 48 ). In the present study, we looked in retinotopically corresponding regions of $\mathrm{V} 2$ and found that an equiluminant sinusoidal red-gray grating of $2.0 \mathrm{c} / \mathrm{deg}$ produced 1.4 times more uptake than a grating that was identical except for a very low $(0.1 \mathrm{c} / \mathrm{deg})$ spatial frequency. For each stimulus region the measurements in V2 were made by measuring peak density in 30 places along the stripes. In both cases, all the stimulus-related uptake was confined to the thin V2 stripes, and an unambiguous retinotopic border could be seen between the V2 regions stimulated by the 2.0 vs $0.1 \mathrm{c} / \mathrm{deg}$ gratings.

Such a result is consistent with the presence of color cells with strong inhibitory surrounds (e.g., double-opponent) in the thin stripes for the following reason. Such cells will presumably respond well to the color grating but not to the spatially diffuse color variations. Single-opponent cells will presumably respond well to both. Therefore, the relative increase in DG uptake in response to the color grating can be rationalized as a stimulation of double- and single-opponent color cell types, as opposed to single-opponent only in response to the spatially diffuse color stimuli. Our faith in this interpretation is shored up by electrophysiological reports of color cells with strong inhibitory surrounds in the V2 stripes (Hubel and Livingstone, 1985).

One unexpected finding that emerged from our DG studies of color in striate cortex is that stimuli of wavelengths near 550 $580 \mathrm{~nm}$ (greenish-yellow through yellow) produced very little uptake compared with wavelengths near the extreme of the spectrum [e.g., $470 \mathrm{~nm}$ (blue) and $610 \mathrm{~nm}$ (reddish-orange)]. Our working interpretation of this DG effect is that the ineffective wavelengths are near enough to the cross-point for red-green cells so that cells are not excited by the $550-580 \mathrm{~nm}$ stimulus. Since these red-green cells are the most common variety of color cell (e.g., Ts'o et al., 1988), this effect presumably swamps the contribution of the blue-yellow cells, which will presumably fire well at $550-580 \mathrm{~nm}$. Such a bias in peak firing to the middle wavelengths can also be seen in a single-unit studies using the same stimulus equipment (Thorell, 1980; Thorell et al., 1984).

Because so much of the input to V2 comes from V1, one would expect that a similar wavelength bias would manifest itself in V2 as well. This was borne out in a number of cases, one of which is shown in Figure 14 (case 38). In relevant visual field regions of this case, the stimulus was a color-varying sinusoidal grating using color-gray pairs. The color-gray pair was adjusted so that the color and gray were approximately equal in luminance. Grating orientation was systematically varied. The spatial frequency of the stimulus $(2.7 \mathrm{c} / \mathrm{deg})$ was low enough so that it produced high uptake in the blobs in striate cortex as well as the thin stripes in V2 (see Fig. 14). The stimulus was divided into 4 radially symmetric sectors, with the lines of division along oblique rays set at $45^{\circ}, 135^{\circ}, 225^{\circ}$, and $310^{\circ}$. In one of the sectors, the grating was red flickered against gray, in another it was (yellowish) green against gray, and in another it was blue against gray. The stimulus in the fourth sector is irrelevant to the present discussion. Uptake was high when produced by wavelength combinations using colors near the spectral extremes (i.e., blue-gray and red-gray) but near-absent when produced by colors near the center of the visible spectrum [e.g., (yellowish) green-gray]. Figure 14 illustrates the difference in effect between the red-gray and yellowish green-gray stimuli.

As we have seen before, black-white gratings of varied spatial frequency, at varied orientations, produce highest uptake in both sets of dark cytox stripes, and moderate uptake in the interstripe regions (see Figs. 3, 6, 7, and 9). Color-varying gratings of lower spatial frequency, and spatially diffuse color stimuli, produce uptake that is essentially confined to the thin stripes (see Figs. 12, 13). From this data one might expect that colorvarying gratings containing higher spatial frequency components (and varied orientations) would produce a pattern of uptake that is a compromise between the 2 patterns described above. The effects of such stimuli are worth examining not only 


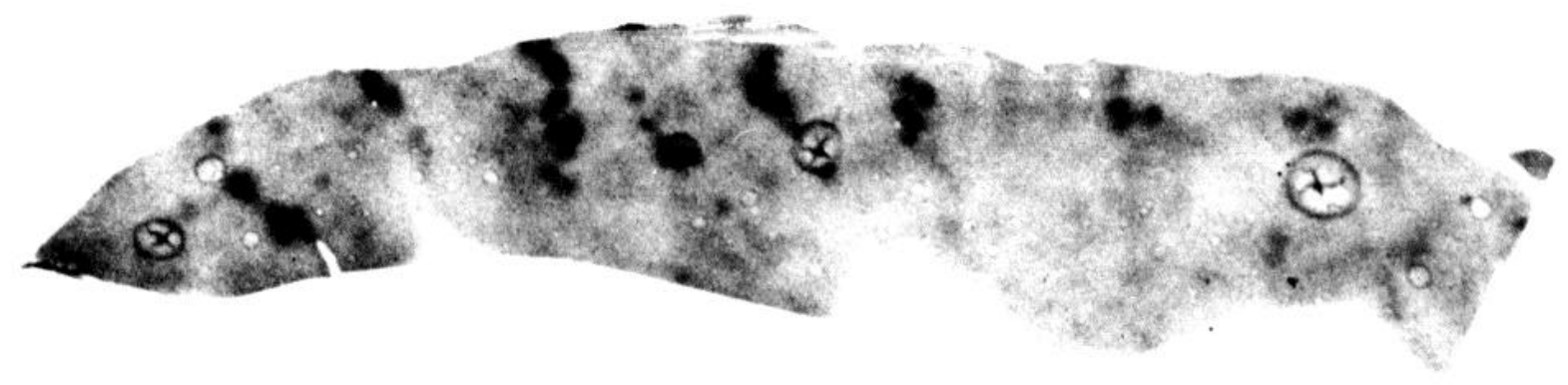

A
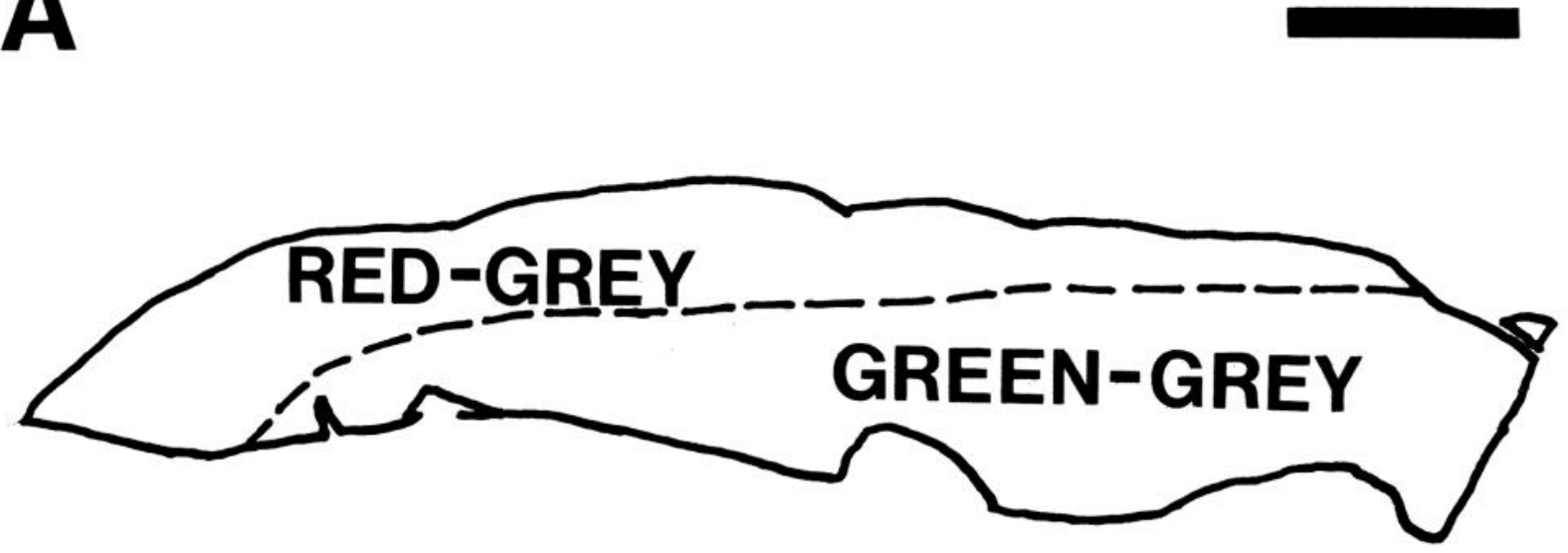

B

Figure 14. Split-field demonstration that wavelengths near the spectral extremes produce more DG uptake than middle wavelengths. A, DG autoradiograph from dorsal V2, cut near-parallel to the cortical layers. The fovea is represented at the right. The visual field that projected to this portion of V2 contained 2 visual stimuli in adjacent portions of the visual field. Both visual stimuli were equiluminant, sinusoidal, color-varying gratings of $2.7 \mathrm{c} / \mathrm{deg}$, presented at varied orientations, drift rates, and directions. One grating was red/gray, and the other was yellowish-green/gray. $B$, Diagram of the representation of the stimulus borders on the cortex. The stimulus was viewed monocularly. Stripes of high DG uptake are produced by the red/gray stimulus: analogous stripes are very faint or absent when produced by the yellowish-green/gray stimulus. Scale bar, 5 $\mathrm{mm}$.

in the interests of thoroughness, but also because they may represent an ecologically more relevant test of color stimuli: most colored stimuli in the natural world are not large regions of color with fuzzy boundaries.

We tested this by stimulating a monkey (case 12) with a squarewave grating of varied spatial frequency $(1,2$, and $4 \mathrm{c} / \mathrm{deg})$ of systematically varied orientations, velocities, and directions. At any given time the grating was made up of equiluminant color pairs of either red-green, blue-yellow, or cyan-purple. The stimulus was viewed binocularly, and the binocular disparity was not varied.

An autoradiograph from this case is shown in Figure 15. Uptake is highest within a thin set of stripes, second highest within what appears to be the thick stripes, and lower (but still above unstimulated level) within the apparent interstripe regions. Overall, this compromise pattern of DG uptake seems more similar to that produced by black-white gratings than to that produced by more spatially diffuse color patterns. However, the high uptake on the thin stripes presumably reflects higher activity in the color cells of these regions, resulting from the use of color gratings. It could be argued that this DG pattern may reflect an artifactual luminance modulation in the grating because even a minor deviation from equiluminance would produce a low-contrast luminance grating when viewed through achromatic magnocellularly biased cortical channels. However, uptake in striate layer $4 \mathrm{~B}$ of this case showed no measurable stimulus-related DG uptake, which indicates that our "equiluminance" value was within a few percent of true equiluminance (Tootell et al., 1988d).

\section{Contrast}

A number of electrophysiological studies have shown that magnocellular LGN cells are much more sensitive to luminance contrast than parvocellular cells (Sperling et al., 1978; Kaplan and Shapley, 1982; Hicks et al., 1983; Derrington and Lennie, 1984). This segregation of contrast sensitivity begins in the retina (Kaplan and Shapley, 1986) and the magnoparvo differences in contrast sensitivity appear to remain largely segregated throughout the striate cortex as well (Tootell et al., 1988d). In the striate cortex, visual stimulation with gratings of contrast below the parvocellular threshold (about $10-12 \%$ ) produces uptake in the magnorecipient $4 \mathrm{Ca}$ but not the parvorecipient $4 \mathrm{Cb}$. The low $(8 \%)$ contrast stimulus also produces moderate uptake in layer $4 \mathrm{~B}$ (which receives major input from $4 \mathrm{Ca}$ ), and lighter labeling in striate layers $2+3$ (which are dominated by parvocellular information).

Most of the input from V1 to V2 comes from striate layers $2+3$. Thus, one would expect that a grating of contrast below 


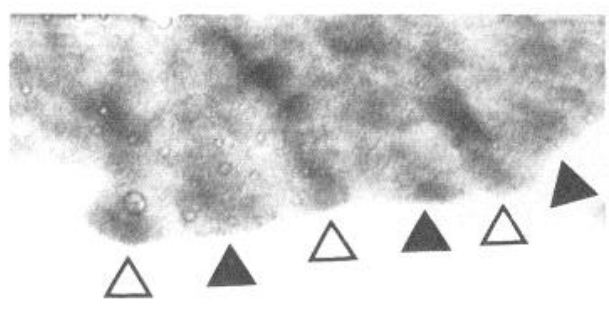

Figure 15. Color gratings include middle and high spatial frequency components produce high DG uptake in thick as well as thin stripes. The autoradiograph here was taken from an animal that was shown, binocularly, a square-wave grating of 1,2 , and $4 \mathrm{c} / \mathrm{deg}$, at varied orientations, drift rates, and directions. The grating was made up of the equiluminant color pairs blue-yellow, red-green, and cyan-purple. Uptake in response to this stimulus is highest in the thin stripes (indicated by hollow triangles), as in cases stimulated by color variations of lower spatial frequency. However, uptake is also reasonably high in the thick stripes (indicated by the filled triangles), as in cases stimulated by blackwhite gratings of otherwise similar spatial characteristics (cf. Fig. 3). Scale bar, $5 \mathrm{~mm}$.

the parvocellular threshold (e.g., $8 \%$ ) would produce relatively little uptake in V2 since it produces little uptake in striate layers $2+3$. However, striate layer $4 \mathrm{~B}$ also projects more faintly to the thick stripes (Livingstone and Hubel, 1987). Thus, we might expect some uptake in the thick V2 stripes, due to input from the moderately active layer 4B.

In experiments with stimulus gratings of $8 \%$ contrast, these expectations were generally met. The stimulus in this case (case 43) was divided into 4 radially symmetric sectors, separated along oblique rays $\left(45^{\circ}, 135^{\circ}\right.$, etc. $)$. In each sector an achromatic grating of varied spatial frequency $(1,2$, and $4 \mathrm{c} / \mathrm{deg})$ appeared, which was moved at a range of velocities in both directions. For reasons that are irrelevant here, orientation was kept constant rather than varied. The only difference between the grating in different sectors was the contrast: different sectors had a contrast of either $8,18,28$, or $38 \%$. The stimulus was viewed monocularly.

In Figure 16 an autoradiograph is shown that includes the representation of the stimulus border between the $8 \%$ and $38 \%$ contrast sectors. Patterns of uptake in response to the $38 \%$ contrast grating are very much like those we described above in response to $\sim 95 \%$ contrast gratings of a single orientation (e.g., Figs. 9-11). However, in response to the $8 \%$ grating, very little uptake is produced in V2. Furthermore, the uptake that does occur in this portion of V2 is generally confined to every other
V2 stripe; this can be seen by comparing the DG autoradiograph in Figure $16 C$ with its cytox-stained counterpart in Figure $16 \mathrm{D}$. For the reasons given above, we take these to be the thick V2 stripes.

\section{Spatial frequency}

In striate cortex, sinusoidal gratings of low spatial frequency (and varied orientation) produce highest uptake in the cytox blobs, and gratings of a high spatial frequency produce highest uptake in the interblobs. This presumably reflects differences in spatial frequency tuning seen in magno- and parvocellular channels at lower levels (Kaplan and Shapley, 1982; Hicks et al., 1983; Derrington and Lennie, 1984). Since HRP evidence indicates that the blobs project to the interstripes (Livingstone and Hubel, 1983), one might expect that gratings of low spatial frequency would produce high uptake in the thin V2 stripes and that high spatial frequencies would produce high uptake in the interstripe regions. Generally, this seems true in our DG data from V2. However, the spatial frequency organization in V2 is somewhat more complicated than one might have expected from the HRP data. On the other hand, it can also be easily reconciled with the HRP data.

To examine the DG patterns in response to low spatial frequencies, we stimulated a monkey (case 20) with a sinusoidal grating of $1 \mathrm{c} / \mathrm{deg}$, binocularly, at orientations that were systematically varied in $45^{\circ}$ steps, and at varied velocities in both directions per orientation. The binocular disparity was varied continuously $( \pm 1 \%)$ during the period of DG uptake.

Figure 17 shows the data from this case. High uptake is confined to parallel stripes running roughly perpendicular to the V $1-\mathrm{V} 2$ border. The spacing between the stripes (about $2 \mathrm{~mm}$ ) is consistent with labeling in every V2 stripe, as opposed to only the thin or thick stripes (see Figs. 5, $C-F, 17 B$ ); every dark DG stripe had a corresponding stripe of dark cytox staining in the same tissue section. We conclude that a low spatial frequency grating produces high uptake not only on the thin stripes (as expected from the projection of the blobs to the thin stripes), but also on the thick stripes as well. Presumably, the activation of the thick stripes arrives by way of striate layer $4 \mathrm{~B}$; blobaligned patches in layer $4 \mathrm{~B}$ also respond robustly to low spatial frequency gratings (Tootell et al., 1988e).

To examine the organization of activity produced by a high spatial frequency grating, we stimulated a different animal (case 19) with a stimulus identical to that described above except that we used a grating of $6.5 \mathrm{c} / \mathrm{deg}$. The grating that was presented at varied orientations (in $45^{\circ}$ steps) moved in both directions and at varied velocities. The stimulus was viewed binocularly, and binocular disparity was varied $\left( \pm 1^{\circ}\right)$.

Figure 16. Within-section comparison of the effects of gratings of medium versus low luminance contrast. $A$, Autoradiograph from ventral V2, cut near-tangential to the layers. The stimulus was arranged so that in one wedge of the visual field, a grating of $8 \%$ luminance contrast was presented. In an adjacent wedge, a grating of $38 \%$ contrast was presented. $B$, Diagram of the representation of each of the stimulus wedges on the cortex. Both stimuli were achromatic, square-wave gratings of varied spatial frequency, drift rate, and direction, but a single (horizontal) orientation. The gratings were viewed monocularly. In the region stimulated by the grating of $38 \%$ contrast, patterns of uptake are as expected from other high contrast, single-orientation cases (see Figs. 9-11). In the region stimulated by the $8 \%$ contrast grating, there is very little uptake at all, except for some minor uptake along every other cytox stripe. This is illustrated most clearly in $C$ and $D$ : $C$, Autoradiograph from a section adjacent to that shown in $A$ (dashed lines indicate the stimulus border); $D$, the position of the thick and thin cytox stripes (based on this and other sections) is illustrated by filled and unfilled triangles, respectively. In comparing $C$ and $D$, it appears that the $8 \%$ contrast grating produces DG uptake mostly in the thick stripes, as one would expect from the striate projections to V2. Scale bar, $5 \mathrm{~mm}$. 

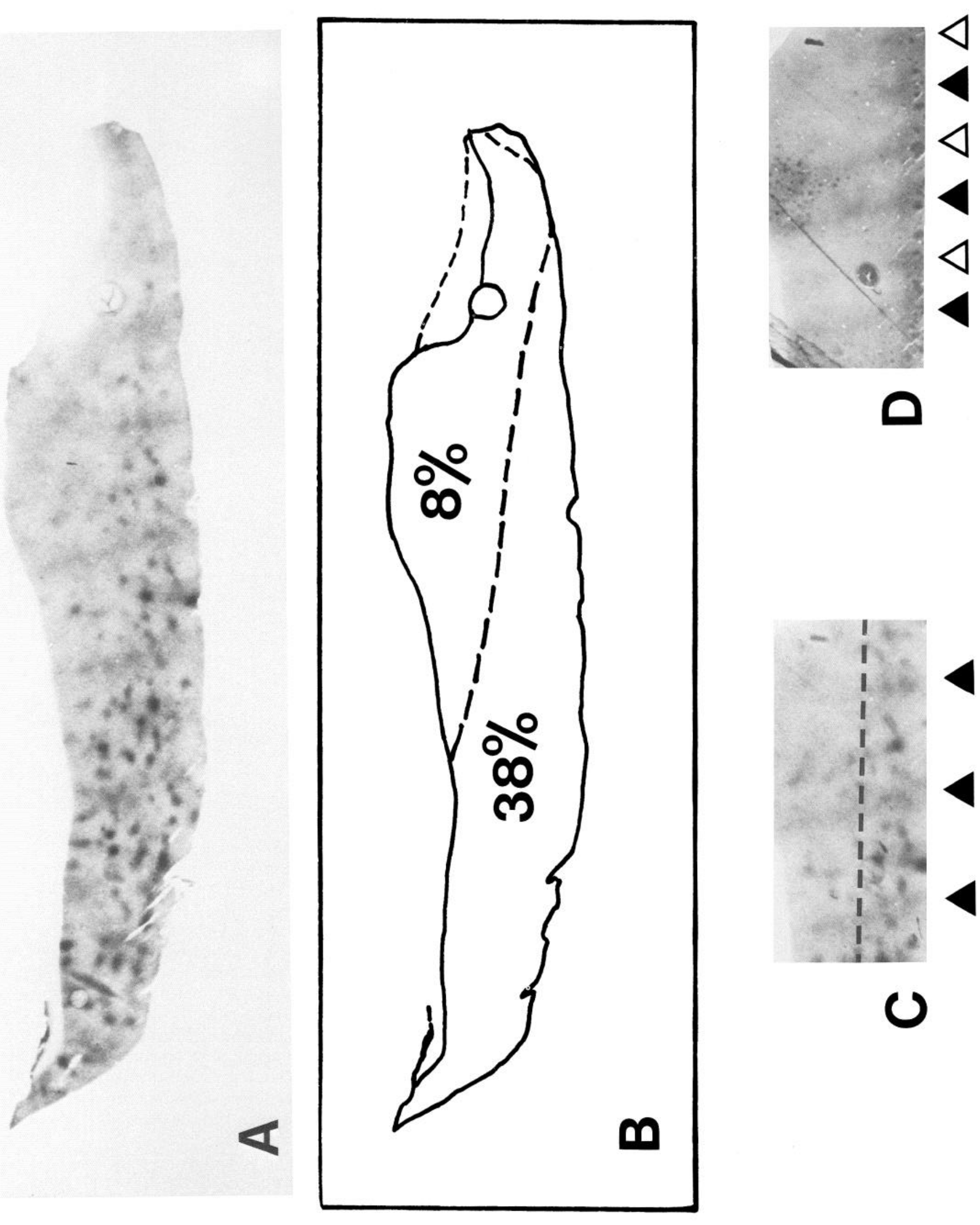
Figure 17. Low spatial frequencies produce high uptake on both sets of dark cytox stripes. $A$, Autoradiograph from dorsal $\mathrm{V} 2 ; B$, same section, after staining for cytox. The stimulus was an achromatic sinusoidal grating of $1 \mathrm{c} / \mathrm{deg}$, presented binocularly at systematically varied orientations, directions, and drift rates. In $A$, high DG uptake is confined to stripes about 2-3 $\mathrm{mm}$ apart, running approximately perpendicular to the V1V2 border near the top of the section. In $B$, all but one of the DG stripes has a dark cytox stripe counterpart. The single DG stripe which has no obvious cytox counterpart, in fact, has one in a deeper section. Figure $5, C-F$ shows DG stripes produced by a similar stimulus, which are more obviously thick and thin. Scale bar, $5 \mathrm{~mm}$.

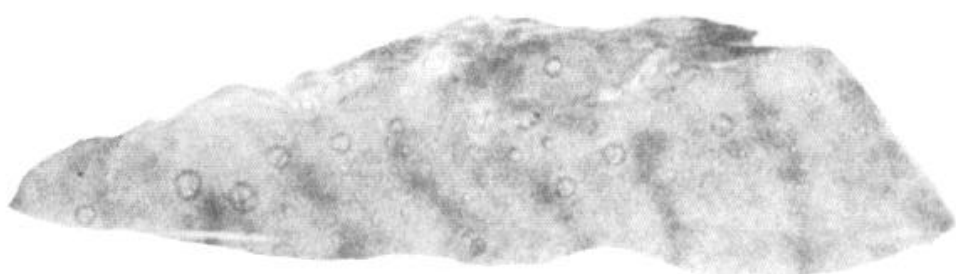

A

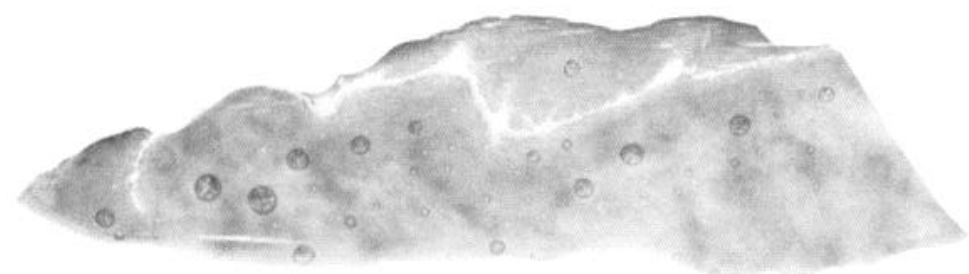

B
Figure 18 shows an autoradiograph from this case; it is typical of results from other cases viewing the same stimulus. The topography is marked by isolated patches (in 3 dimensions, isolated columns) separated by $1-1.7 \mathrm{~mm}$, with little or no labeling of either set of dark cytox stripes. In some cases (e.g., Fig. 19, $B, I)$, and in a preliminary report (Tootell et al., 1983), DG labeling in response to this stimulus includes every other stripe; but with a larger sample, this has turned out to be the exception rather than the rule. Of the 9 cases we could examine that were stimulated with a high spatial frequency grating, V2 stripes (in addition to the isolated columns) were labeled in 2 cases, were absent in 5 cases, and were ambiguous in 1 case.

In some cases stimulated by high spatial frequency gratings, the isolated patches appear to be positioned without regard to the positions of the cytox stripes throughout area V2. However, when cytox histology does show stripes, most of the isolated patches are positioned in the V2 interstripes, the regions of light cytox staining (see Fig. 19). Within a given interstripe region, they are spaced apart from one another at a relatively regular distance. Presumably, the array of isolated patches often does not look as regular in the absence of the cytox stripe landmarks because the patches are sometimes not aligned in rows perpendicular to the stripes and interstripes and because there is sometimes high uptake in one set of cytox stripes (e.g., Fig. 19, B, $H)$.

In order to be certain that the spatial frequency differences in DG topography were real, we did a split-field experiment. In this case (case 55), the visual stimulus was divided in half, along the horizontal meridian. In both halves, the stimulus was an achromatic, sinusoidal grating of varied orientations, directions, and velocities. In the bottom half, the grating had a spatial frequency of $1 \mathrm{c} / \mathrm{deg}$, and in the top half it was $6.5 \mathrm{c} / \mathrm{deg}$. The stimulus was viewed binocularly, and disparity was not varied.

The effects of the 2 stimulus halves can be compared in Figure 20. Figure 20 is taken from a single tissue section. In Figure
$20 \mathrm{~A}$ (dorsal V2), the low spatial frequency grating produced a pattern of high uptake in stripes in apparently every stripe. In Figure $20 B$ (ventral V2), the high spatial frequency grating produced a pattern of isolated patches, as described above. Because the 2 different DG patterns are so different from one another, and because they are consistent with those seen earlier in between-animal comparisons, we conclude that there is a functional organization in V2 that can be tapped by changing only the spatial frequency of sinusoidal gratings. Whether "spatial frequency" remains the most accurate description of these functional differences is yet to be determined.

\section{Discussion}

The study of cortical organization is important for a number of reasons. Since brain science is still in its infancy, it is easy to justify the demonstration of a new (and often beautiful) structure or organization purely on the basis of "look-see" natural history - in other words, for reasons of classical anatomy.

However, there is also a more incisive value to the study of cortical organization. It has become increasingly clear that in cortical areas where stimulus parameter " $X$ " first becomes a significant functional variable, there is often a functional organization of cells along those same lines. For example in the striate cortex, where cells first become orientation selective and where input from the 2 eyes is first joined, orientation and ocular dominance columns appear. In area MT, where cells for the first time become almost uniformly direction-selective, directional columns appear.

Historically, a single-unit selectivity for parameter " $\mathrm{X}$ " has usually been noticed before a cortical organization for parameter " $\mathrm{X}$ " has been tested, but this need not be the case. For instance, one can imagine that if orientation sensitivity had not yet been found in individual cells of striate cortex, that orientation $\mathrm{col}$ - 

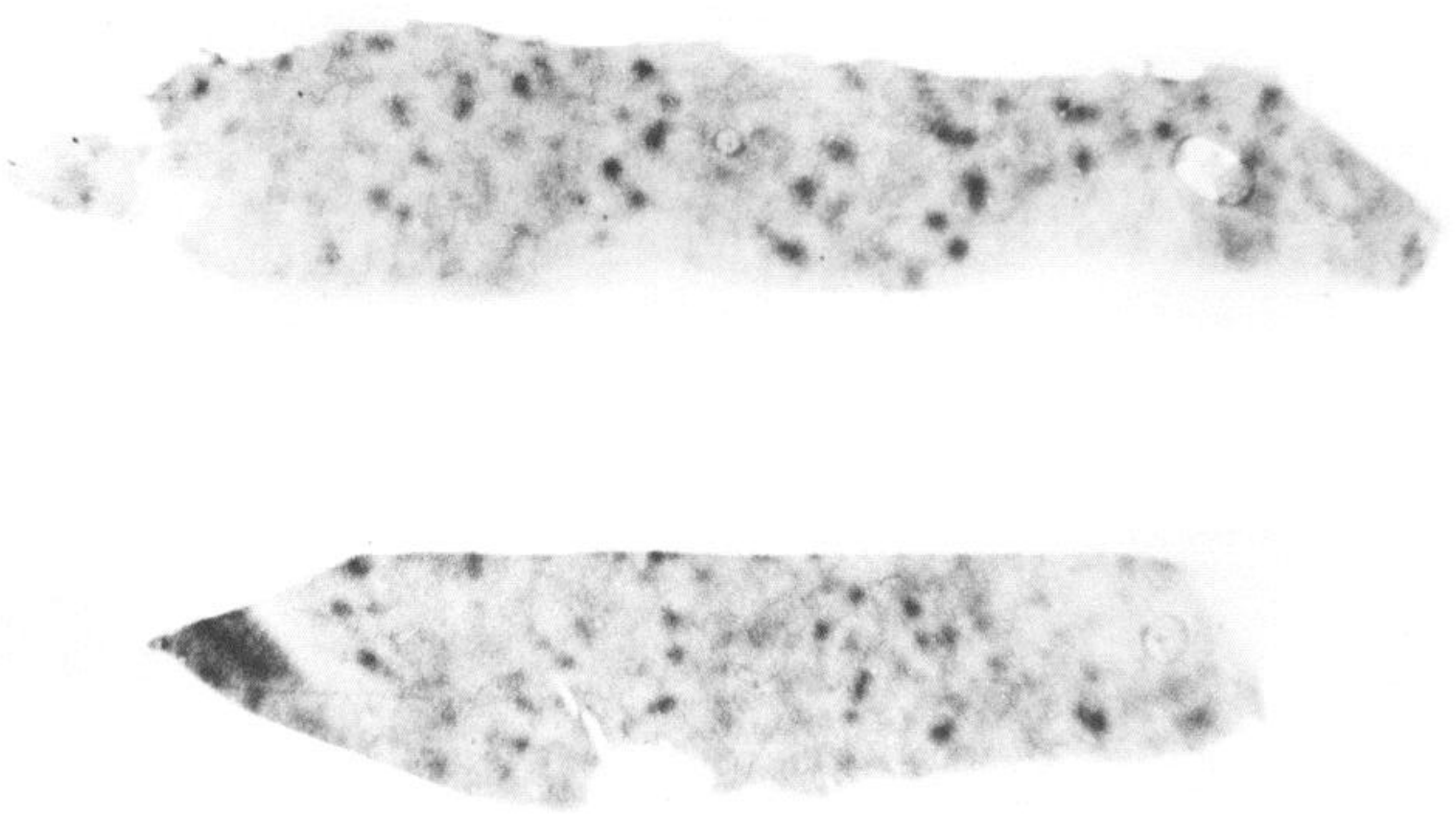

Figure 18. Representative topography of DG uptake in response to a high spatial frequency grating. The figure shows an autoradiograph from dorsal and ventral V2, cut near-tangential to the cortical layers. The stimulus was an achromatic grating of $6.5 \mathrm{c} / \mathrm{deg}$, shown binocularly at varied orientations, directions, and drift rates. Such a stimulus produces a pattern of isolated columns throughout V2 (as illustrated here) and (more rarely) high uptake in one set of stripes as well (see Fig. 18). Scale bar, $5 \mathrm{~mm}$.

umns might still be found (either serendipitously or by deliberate test) by experimenters working with different stimuli, including bars and gratings, in DG or optical recording experiments. In such a hypothetical case, the demonstration of orientation columns would presumably motivate follow-up single-unit experiments aimed at clarifying the nature of these strange, unexpected orientation-specific anatomical groupings. The point here is that studies of the cortical organization per se can potentially lead the way to clarifying the essential functions of a given cortical area rather than follow.

This point is important to keep in mind when considering the present results from area V2. Compared with V1, there have been comparatively few electrophysiological studies of the properties of cells in V2. Thus, DG studies in V2 are not directed towards simple confirmation of electrophysiological data about compartmentalization of function, as they often are in V1. In the present study of $\mathrm{V} 2$, we found that it was much more common to test the DG effects of a certain stimulus versus its appropriate control, and to find the presence or absence of a DG organization, all without benefit of a background of extensive electrophysiological evidence. In area V1, almost all the electrophysiological evidence for functional organizations has found DG confirmation, and the converse has seemed true as well. Thus, we expect that even the more unexpected DG results from the present study will eventually find electrophysiological confirmation, rather than turning out to be some strange metabolic epiphenomenon.

\section{Baseline conditions}

In V1, stimulation with either a spatially diffuse gray, a spatially diffuse black-vs-white, or a completely dark stimulus produces negligible uptake in either the V1 blobs or the interblobs. The situation is similar in V2: such stimuli produce little or no increase in uptake over unstimulated levels, in either the stripes or the interstripes.

However, if we consider the pattern of DG uptake in response to very general, spatially patterned stimuli (such as gratings of variable spatial frequency at varied orientations), the parallel between results in V1 and V2 is less exact. When viewed binocularly, such stimuli produce a relatively uniform pattern of uptake in V1 at parafoveal eccentricities, and relatively higher uptake in the blobs near the fovea. In V2, however, uptake in response to such stimuli is always higher in the dark cytox stripes than in the interstripes. From the DG data in parafoveal V1, and from the known connections between blobs and stripes in V2, one might have expected a pattern of DG uptake in parafoveal V2 which was uniformly distributed across the stripes and interstripes. Such a DG result does not occur.

In retrospect, it is probably overly simplistic to have expected it. There is no a priori reason to expect that the ratio of uptake in the upper-layer blobs and interblobs will be exactly related to that in their efferent targets, the stripes and interstripes. Much of the discrepancy may be due to the presence of strong endstopping in interstripe cells (cf. Figs. 3 and 4; see also Livingstone and Hubel, 1987). Furthermore, the stripes and interstripes get input from other cortical and subcortical areas, in addition to those from the striate cortex (e.g., Curcio and Harting, 1978; Wong-Riley, 1979; DeYoe and Van Essen, 1985). Nonetheless, it is somewhat paradoxical that in response to a very general visual stimulus, DG uptake is consistently higher in the dark cytox V2 stripes, though the stripes often stain only irregularly or faintly in a cytox reaction. In parafoveal area V1, the opposite 

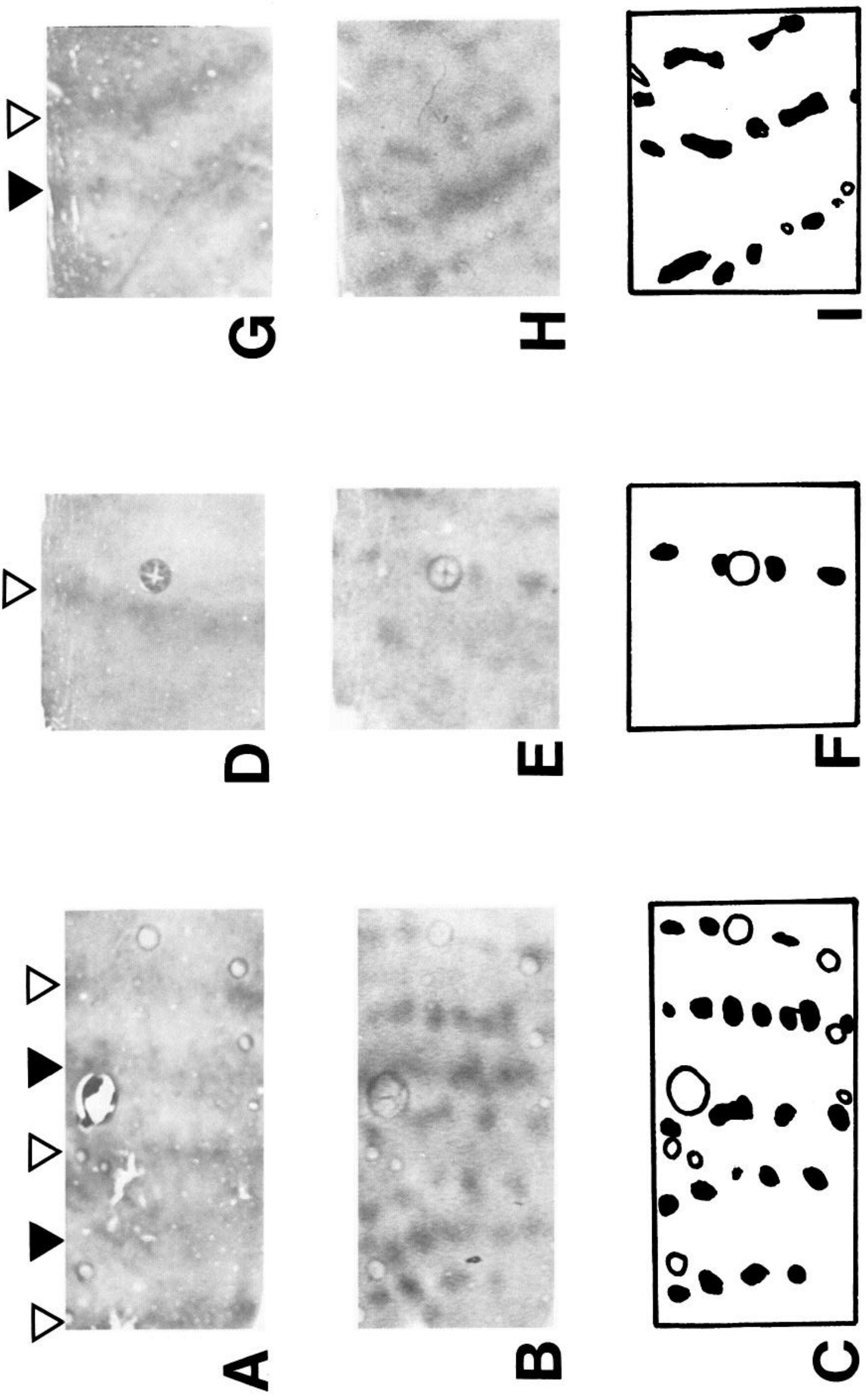

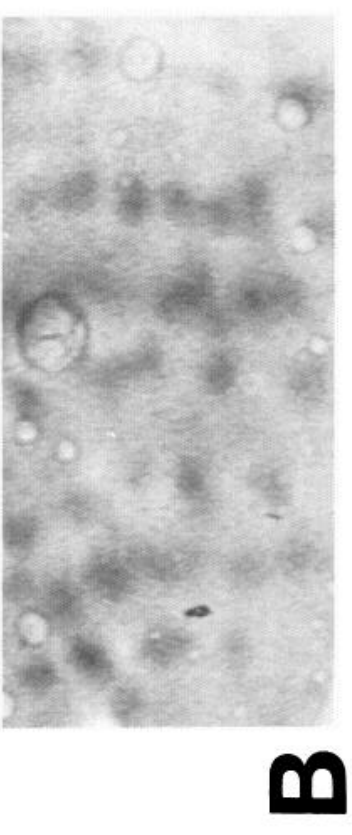

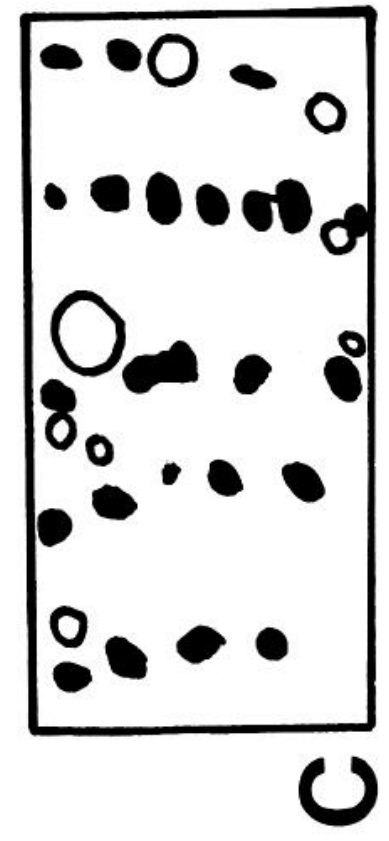

4

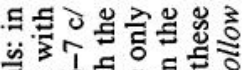

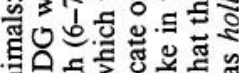

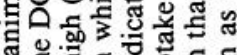

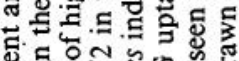

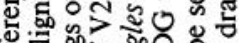

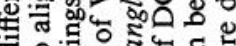

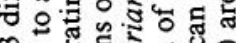

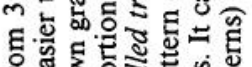

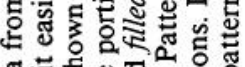

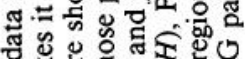
ชิ

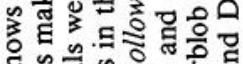

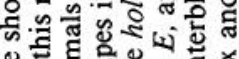

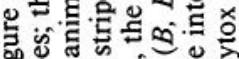

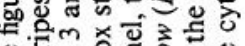

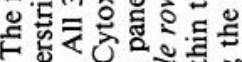
잉.

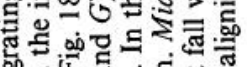
ช. 究 可位政

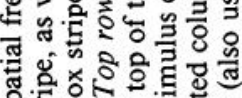
की

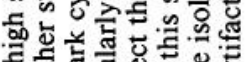
๙

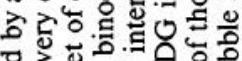

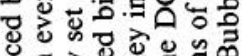
త

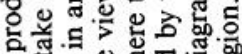

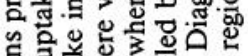

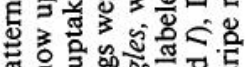

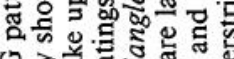

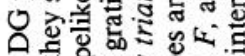

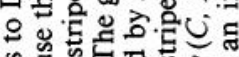
记

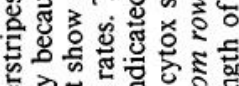

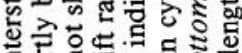

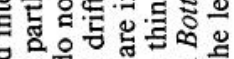

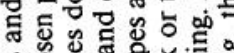

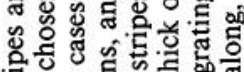

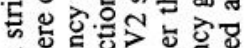

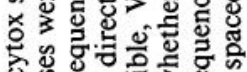

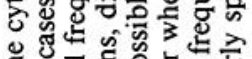
⿷匚⿳丨コ丨

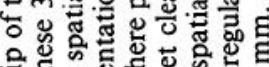

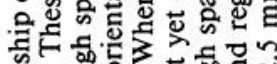

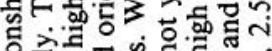

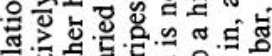
ฮ

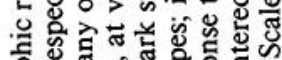

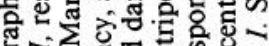
额

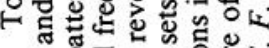

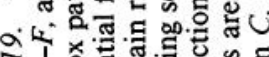

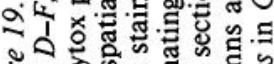

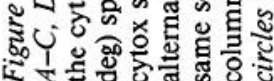



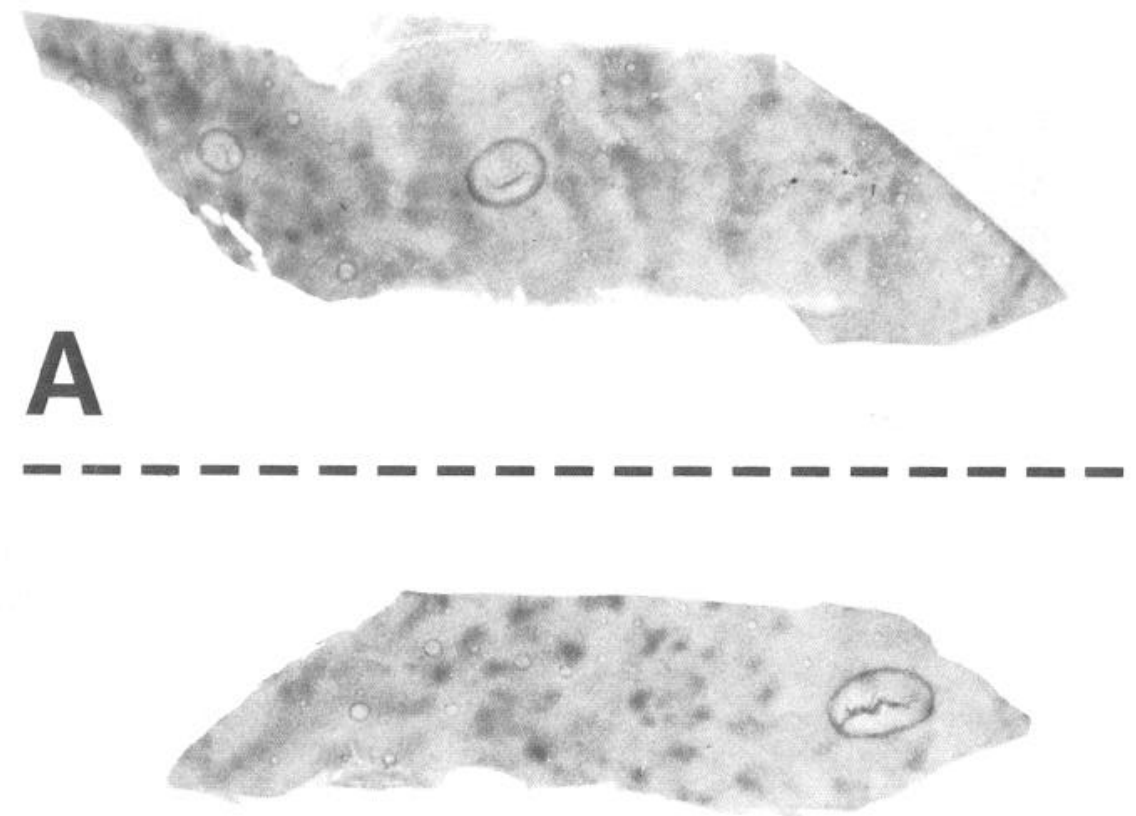

B
Figure 20. Split-field test of the effects of low versus high spatial frequency gratings. $A$ and $B$, Autoradiographs from dorsal and ventral V2, respectively. The fovea is represented to the left in each section, and more peripheral eccentricities are mapped towards the right. In the visual field region corresponding to $A$, the animal viewed a grating of $1 \mathrm{c} / \mathrm{deg}$; in $B$, a $6.5 \mathrm{c} / \mathrm{deg}$ grating. In both halves, the stimulus was otherwise identical. It was shown binocularly, at varied orientations, directions, and drift rates. The low versus high spatial frequency gratings produced obviously different patterns of uptake, and these differences are as one would predict from similar DG comparisons between different animals. A high spatial frequency grating produces high uptake in isolated columns (aligned in rows in the interstripes), and a low spatial frequency grating produces high uptake in both sets of dark cytox stripes. Scale bar, 5 $\mathrm{mm}$. is the case: blobs stain consistently darker than interblobs; DG uptake in response to a very general stimulus is distributed fairly uniformly across the blob versus interblob regions. Thus, the correlation between cytox activity and DG uptake in response to a very general stimulus is poor (and discrepant in opposite ways) in areas V1 and V2.

In cytox-stained material, the dark "stripes" can often be described more accurately as a string of patches (e.g., Fig. 5). In the DG material, such patches are even more obvious in response to a number of different types of visual stimuli, and they extend above and below layers $3 \mathrm{~B} / 4$ as columns. Though a patchiness of the cytox-stained stripes has been mentioned previously (Horton, 1984; Livingstone and Hubel, 1984; WongRiley and Carroll, 1984), there is only sparse data on the degree of correspondence between the patches and afferent or efferent connections to the V2 "stripes" (DeYoe and Van Essen, 1985). Nor is there any good information on the functional organization of the patches versus the intervening gaps between the patches. Such data may be the next critical level of analysis in understanding the architecture of $\mathrm{V} 2$.

\section{Retinotopy}

In the first comparison of retinotopic patterns in $\mathrm{V} 1$ and $\mathrm{V} 2$ (Fig. 6), DG patterns are fainter in layers $3 \mathrm{~B} / 4$ of $\mathrm{V} 2$ than they are in the striate cortex. Thus, it is possible we have underestimated the retinotopic spread in this particular case $(\sim 400$ $700 \mu \mathrm{m}$, half-amplitude) relative to that in striate cortex layers $2+3(250-500 \mu \mathrm{m})$ because of a "tip of the iceberg" problem. This is especially true considering that the retinotopic borders produced by a different, much larger visual stimulus (Fig. 7) are blurrier in the same layer of V2. On the other hand, it is possible that the small blinking checks used in the Figure 6 stimulus stimulate only a subpopulation of cells in V2 layer 4, each with small receptive fields: this would account for both the discreteness of the borders and the faintness of the DG patterns in this case. This idea is also supported by the fact that in the case illustrated in Figure 6 (but not in Fig. 7), stimulus-induced uptake does not occur in the upper and lower layers of the striate cortex when stimuli are small, relative to the striate receptive fields (Tootell et al., 1988b).

It would be interesting to know more about the relative positions of receptive fields in thin, thick, and interstripe zones, and the "scalloped" appearance of the retinotopic border in Figure 7 is intriguing in this regard. However, at present we do not have enough DG material to come to solid conclusions.

\section{Ocularity}

In single-unit recordings, cells are strongly monocular in the striate cortical input layers, but they are progressively more binocular in the upper striate layers. In area V2, some cells have been described as having ocular biases, but there are few, if any, strictly monocular cells (Hubel and Wiesel, 1970; Clarke et al., 1976; Baizer et al., 1977; Poggio and Fischer, 1977; Hubel and Livingstone, 1987). From the single-unit evidence one could construe a gradual shift from monocular to binocular, from the striate input layers through the upper layers through V2.

In many respects, the DG evidence indicates a more discrete, step-wise transition between a monocular organization and a binocular one. The ocular dominance stripes that appear in layer $4 \mathrm{C}$ extend through all striate layers, with very little cross-activation of the unstimulated eye dominance stripes (Kennedy et al., 1975; Tootell et al., 1988a). In the DG, the transition between the monocularly segregated architecture and an ocularly mixed architecture appears to be in the connection between striate layers $2+3$ and layers $3 B / 4$ of V2. Further work may conceivably demonstrate an ocular dominance architecture in 
layers $3 \mathrm{~B} / 4$ of $\mathrm{V} 2$, but the preliminary evidence on this point is negative. In particular, it does not look as if half of the layers $3 \mathrm{~B} / 4$ patches receive input from one eye and half from the other eye (Ts'o et al., 1988).

In other respects, the DG patterns do match the single-unit reports on ocularity. In particular, the borders of the ocular dominance stripes in V1 are fuzzy in many extragranular layers, so onc can rationalize the presence of binocular cells ncar the (fuzzy) borders of the DG ocular dominance stripes.

Hubel and Livingstone (1987) have reported that groups of disparity-specific, binocular-only cells are common in the thick stripes. We have not yet designed any DG experiment to address this question directly, but such a disparity-specificity is not obvious in the present DG results. For instance, in binocular cases stimulated al a fixed disparity, stimulus-induced uptake was often continuous within the thick stripes rather than patchy. Second, robust uptake could be produced within the thick stripes even with monocular stimulation (see Fig. 7), at least in layer 4. It will probably be necessary to use optical or double-label DG techniques to resolve this issue completely.

\section{Orientation}

Among the most straightforward of DG results is the demonstration of orientation columns in V2. The split-field test proved that the DG columns are related to orientation per se, rather than to some attendant stimulus variable. In the monkey, the anatomical evidence for orientation columns in areas beyond $V_{1}$ is scant (but see Livingstone and Hubel, 1982), although orientation tuning and gradual shifts in orientation tuning have been reported in electrophysiological studies of V2 (DeYoe and Van Essen, 1985; Shipp and Zeki, 1985; Hubel and Livingstone, 1987) and V3 (Van Essen and Zeki, 1978). Orientation columns have been reported in areas V1 and V2 of the cat (Hubel and Wiesel, 1962; Matsubara et al., 1986), which are presumably homologous to areas V1 and V2 of the monkey. The fact that orientation columns exist in at least the first 2 visual cortical areas underscores the importance of orientation selectivity in the analysis of visual information in the cortex.

By the very simplest of models, the bandwidth of orientation selectivity in cells should be directly related to the width of DG uptake in orientation columns, relative to the repeat distance for a full cycle of orientations. For instance, if all cells in a given area were suddenly made much more selective for the orientation of stimuli (that is, if the orientation tuning curves had smaller bandwidths), then the DG orientation columns would presumably be correspondingly skinnier. If cells became much less selective (for instance, almost nonoriented), then DG uptake in response to an oriented pattern would be much more uniform across the cortex; the orientation "columns" would be correspondingly broadened.

In the monkey, the orientation selectivity of cells in area $\mathrm{V} 1$ is similar to that in area V2. In V1, the average bandwidth for oriented cells is about $55^{\circ}$ (half-amplitude, full width) (Schiller et al., 1976; De Valois et al., 1982), and in area V2 it has been reported to be similar (Baizer et al., 1977). Given the electrophysiological data on the orientation bandwidths, columns in $\mathrm{V} 1$ and V2 should be about equally wide in these 2 areas, as seen in the simple model of DG orientation columns described above. In densitometric measurements, the DG orientation columns in area V2 are very close in width to those in V1. However, there are about 1.6 times as many orientation "columns" per unit surface area in V1 as in V2 $\left(2.4\right.$ columns $/ \mathrm{mm}^{2}$ vs 1.5 columns/ $\mathrm{mm}^{2}$, respectively).

The DG differences between V1 and V2 show up not only in straightforward counts but also in double-label DG results from stimulation with gratings of 2 orthogonal orientations. In $\mathrm{V} 1$, such stimuli produce 2 patterns of uptake without intervening gaps, but in V2, the orientation columns produced by both orthogonal orientations are clearly separated from each other (Tootell et al., unpublished observations). One is therefore left wondering about the spaces between the orthogonal orientation columns in V2: Do they simply represent cells tuned to orientations between the 2 stimulus orientations? This would imply a narrower bandwidth of tuning in the cells (and narrower orientation columns) in V2, and this runs counter to the available electrophysiology. One possible explanation is that cells responding well to the oriented gratings are interspersed with cells of another type altogether (e.g., color-specific, nonoriented, or end-stopped) and that the latter cells form the spaces between columns in the 2-orientation experiments. Again, double-label DG or optical recording techniques may be necessary to clarify this issue.

Color

In most respects, the DG results from equiluminant color stimulation in V2 are consistent with the organization of color cells in V1, and with the known projections from V1 to V2 (Livingstone and Hubel, 1983; DeYoe and Van Essen, 1985; Hubel and Livingstone, 1985). Single-opponent cells are found in the V1 blobs, and the blobs project to the thin V2 stripes. Thus, it is not surprising that spatially diffuse variations in color, which stimulate single-opponent color cells, produce high DG uptake confined to the thin V2 stripes. Such spatially diffuse color stimuli produce negligible uptake in striate layer 4B. Consequently, the weak projection from striate layer $4 \mathrm{~B}$ to the thick stripes of V2 is presumably silent, and this undoubtedly helps confine DG activity to the thin stripes in such cases.

The V1 blobs project only to layers $3 \mathrm{~B}$ and 4 of the $\mathrm{V} 2$ thin stripes. The fact that spatially diffuse color variations produce high uptake in all layers of the thin V2 stripes strongly implies that some single-opponent color cells are found not only in the striate-recipient layers $3 \mathrm{~B}$ and 4 but also in layers above and below it. This idea is strongly supported by the fact that spatially diffuse color variations also produce some color-specific patterns of DG uptake in area V4 (unpublished observations), which in turn receives a projection from cells in the upper layers of the V2 stripes (DeYoe and Van Essen, 1985; Shipp and Zeki, 1985). Thus, there appears to be a preservation of single-opponent color activity from the V1 blobs to layers $3 \mathrm{~B}$ and 4 of the thin stripes, which is then relayed to the upper layers of the thin stripes, and from there to V4 and beyond.

In other respects, DG results in V2 seem to follow directly from those in V1, without obvious surprises. With few exceptions, cells in the upper layers of V1 will not respond to spatially diffuse variations in luminance (Dow, 1974), and DG uptake is correspondingly unaffected by such a stimulus (Tootell et al., 1988c). So it is not surprising that spatially diffuse luminance variations produce little DG uptake in V2 as well as V1. Similarly, the lack of DG uptake in response to middle wavelengths in V1 is translated forward; such wavelengths also produce little or no uptake in V2. (Such a lack of uptake is not a logical necessity, however, because this kind of information could conceivably reach V2 by way of the pulvinar or other routes.) Fi- 
nally, we see DG evidence for surround inhibition in the color cells of the thin V2 stripes. Presumably this is the DG reflection of the surround inhibition reported earlier in single-unit studies (Hubel and Livingstone, 1985).

\section{Contrast}

The results of tests with low-contrast stimuli are also in general accord with the known projections from V1 to V2. When the luminance contrast of a grating was low enough (e.g., $8 \%$ ) so that only the magnocellular (but not the parvocellular) pathway was stimulated in V1, uptake in V2 was confined to every other stripe in V2 (see Fig. 16). Presumably these are the thick stripes, which receive input from layer $4 \mathrm{~B}$ of $\mathrm{V1}$, for the following reason. In response to an $8 \%$ contrast grating, layer $4 \mathrm{~B}$ of striate cortex shows fairly good activation and the upper striate layers show very little (Tootell et al., 1988d). Presumably, then, the DG uptake in the thick stripes in V2 is mediated by the projection from layer $4 \mathrm{~B}$, in isolation from the relatively silent pathway from layers $2+3$ to the interstripes and thin stripes.

\section{Spatial frequency}

In V1, sinusoidal luminance gratings of low spatial frequency produce higher uptake in columns running through the upperlayer blobs, and gratings of high spatial frequency produce columns of higher uptake in alignment with the upper-layer interblobs (Tootell et al., 1988e). At least in the upper striate layers, these differences have now been confirmed with optical techniques (Tootell and Blasdel, 1987).

In V2, low spatial frequency gratings produce high uptake in both the thin and the thick stripes. The high uptake in the thin stripes presumably reflects activity present in the blob-to-thinstripe projection.

The source of activation for the thick V2 stripes is less straightforward to interpret. A projection from striate layer $4 \mathrm{~B}$ has been shown to the thick stripes (Livingstone and Hubel, 1987). However, visual stimulation with a low spatial frequency grating produces high uptake only in the blob-aligned portions of layer $4 \mathrm{~B}$, not throughout the whole layer. This raises the possibility that only the blob-aligned portions of $4 \mathrm{~B}$ project to the thick stripes and that interblob-aligned portions of $4 \mathrm{~B}$ do not. This conjecture is also supported by results from high-frequency stimulation. High spatial frequency gratings produce uptake only in the interblob-aligned portions of $4 \mathrm{~B}$, and these stimuli often do not produce uptake in the thick V2 stripes. Such a subdivision in the layer $4 \mathrm{~B}$ projection may have been missed in the initial HRP data because the projection is relatively faint.

Similar questions arise when we consider the DG pattern produced by high spatial frequency gratings in layers $2+3$. According to the HRP data, the interblobs project to the interstripes (Livingstone and Hubel, 1983). However, high-frequency gratings, which produce uptake within the whole interblob area in V1, produce high uptake in only a subset of the interstripe regions of $\mathrm{V} 2$.

There are at least 2 possible ways in which the HRP results can be reconciled with the DG data. One (obvious) possibility is that end-stopped and non-cnd-stopped cclls, which are found in roughly equal portions in the interblobs (Hubel and Livingstone, 1987), are grouped together into end-stopped and nonend-stopped patches within the interstripes and, further, that the nonstopped cells are tuned to relatively high spatial frequencies.

Another possibility involves a reinterpretation of the original
IIRP data. The original HRP injections were made blindly in $\mathrm{V} 2$, and some of those injections presumably landed within those portions of the interstripes that receive input from the interblobs. The conclusion that the interblobs projected to the (whole of) the interstripes may have been based on those injections. Other injections that landed in the interstripes, but did not produce label in the interblobs, may have been discounted as "bad" injections. The size of the HRP injections $(\sim 150 \mu \mathrm{m})$ is much smaller than the average distance between high spatial frequency DG periodicities $(\sim 1000-1700 \mu \mathrm{m})$. Thus there is plenty of room to suppose that the interblobs project to only a subset of the total interstripe area, which is consonant with the data from high spatial frequency cases.

In the present DG results from $\mathrm{V} 2$, there are a number of unresolved questions. Here we can only appeal to the rhetoric at the beginning of the discussion: the DG at least points to some intercsting architcctural features which will probably need to be resolved by resort to other mapping techniques. In the case of the spatial frequency differences, it is worth emphasizing at least that a cortical distinction based on stimulus size is passed on in a segregated fashion from V1 at least to V2. Single-unit studies will undoubtedly be necessary to clarify the nature of these and other DG differences.

\section{References}

Albright, T. D., R. Desimone, and C. G. Gross (1984) Columnar organization of directionally selective cells in visual area MT of the macaque. J. Neurophysiol. 51: 16-31.

Allman, J. M., and J. H. Kass (1974) The organization of the secondtier visual area (VII) in the owl monkey: A second order transformation of the visual hemifield. Brain Res. 76: 247-265.

Baizer, J. S., D. L. Kobinson, and B. M. Dow (1977) Visual responses of area 18 neurons in awake, behaving monkey. J. Neurophysiol. 40. 1024-1037.

Blasdel, G. G., and G. Salama (1986) Voltage-sensitive dyes reveal a modular organization in monkey striate cortex. Nature 321:579-585.

Clarke, P. G. H., I. M. L. Donaldson, and D. Whitteridge (1976) Binocular visual mechanisms in cortical areas I and II of the sheep. J. Physiol. (Lond.) 256: 509-526.

Curcio, C. A., and J. K. Harting (1978) Organization of pulvinar afferents to area 18 in the squirrel monkey: Evidence for stripes. Brain Res. 143: 155-161.

Derrington, A. M., and P. Lennie (1984) Spatial and temporal contrast sensitivities of neurons in later geniculate nucleus of macaque. $J$. Physiol. (Lond). 357: 219-240.

De Valois, R. L., D. G. Albrecht, and L. G. Thorell (1982) Spatial frequency selectivity of cells in macaque visual vortex. Vision Res. 22: 545-559.

DeYoe, E. A., and D. C. Van Essen (1985) Segregation of efferent connections and receptive field properties in visual area V2 of the macaque. Nature 317: 58-61.

Dow, B. M. (1974) Functional classes of cells and their laminar distribution in monkey visual cortex. J. Neurophysiol. 37: 927-946.

Foster, K. H., J. P. Gaska, M. Nagler, and D. A. Pollen (1985) Spatial and temporal frequency selectivity of neurones in visual cortical areas $\mathrm{V} 1$ and V2 of the macaque monkey. J. Physiol. (Lond.) 365: $331-$ 363.

Gattass, R., C. G. Gross, and J. H. Sandell (1981) Visual topography of V2 in the macaque. J. Comp Neurol. 201: 519-539.

Grinvald, A. E., E. Lieke, R. D. Frostig, C. D. Gilbert, and T. N. Wiesel (1986) Functional architecture revealed by optical imaging of intrinsic signals. Nature 324: 361-364.

Hendrickson, A. E., and J. R. Wilson (1979) A difference in $\left[{ }^{14} \mathrm{C}\right]$ deoxyglucose autoradiographic patterns in striate cortex between $\mathrm{Ma}$ caca and Saimiri following monocular stimulation. Brain Res. 170: 353-358.

Hicks, T. P., B. B. Lee, and T. R. Vidyasagar (1983) The response of cells in macaque lateral geniculate nucleus to sinusoidal gratings. $J$. Physiol. (Lond.) 337: 183-200.

Horton, J. C. (1984) Cytochrome oxidase patches: A new cytoarchi- 
tectonic feature of monkey cortex. Phil. Trans. R. Soc. London [Biol.] 304: 199-253.

Horton, J. C., and D. H. Hubel (1981) Regular patchy distribution of cytochrome oxidase staining in primate visual cortex of macaque monkey. Nature 292: 762-764.

Hubel, D. H., and M. S. Livingstone (1985) Complex-unoriented cells in a subregion of primate area 18. Nature $315: 325-327$.

Hubel, D. H., and M. S. Livingstone (1987) Segregation of form, color and stereopsis in primate area 18. J. Neurosci. 7: 3378-3415.

Hubel, D. H., and T. N. Wiesel (1962) Receptive fields, binocular interaction and functional architecture in cat's visual cortex. J. Physiol. (Lond.) 160: 105-154.

Hubel, D. H., and T. N. Wiesel (1968) Receptive fields and functional architecture of monkey striate cortex. J. Physiol. (Lond.) 195: 215243.

Hubel, D. H., and T. N. Wiesel (1970) Cells snesitive to binocular depth in area 18 of the macaque monkey cortex. Nature 225:41-42.

Hubel, D. H., and T. N. Wiesel (1974) Uniformity of monkey striate cortex: A parallel relationship between field size, scatter and magnification factor. J. Comp. Neurol. 158: 295-306.

Hubel, D. H., T. N. Wiesel, and M. P. Stryker (1978) Anatomical demonstration of orientation columns in macaque monkey. J. Comp. Neurol. 177: 361-380.

Humphrey, A. L., L. C. Skeen, and T. T. Norton (1980) Topographic organization of the orientation columns in the striate cortex of the tree shrew (Tupai glis) II. Deoxyglucose mapping. J. Comp. Neurol. 192: 549-566.

Kaplan, E., and R. M. Shapley (1982) X and Y cells in the lateral geniculate nuclcus of macaque monkey. J. Physiol. (Lond.) 330: 125143.

Kaplan, E., and R. M. Shapley (1986) The primate retina contains two types of ganglion cells with high and low contrast sensitivity. Proc. Natl. Acad. Sci. USA 83: 2755-2757.

Kennedy, T. C., M. H. Des Rosiers, M. Reivich, F. Sharpe, and L. Sokoloff (1975) Mapping of functional neural pathways by autoradiographic survey of local metabolic rate with $\left[{ }^{14} \mathrm{C}\right]$ deoxyglucose. Science 187: 850-853.

Krubitzer, L. A., and J. H. Kaas (1987) Connections of modular subdivisions of cortical visual areas 17 and 18 with the middle temporal area, MT, in squirrel monkey. Soc. Neurosci. Abstr. 13: 3.

Livingstone, M. S., and D. H. Hubel (1982) Thalamic inputs to cytochrome oxidase-rich regions in monkey visual cortex. Proc. Natl. Acad. Sci. USA 79: 6098-7101.

Livingstone, M. S., and D. H. Hubel (1983) Specificity of corticocortical connections in monkey visual system. Nature 304: 531-534.

Livingstone, M. S., and D. H. Hubel (1984) Anatomy and physiology of a color system in primate visual cortex. J. Neurosci. 4: 309-356.

Livingstone, M. S., and D. H. Hubel (1987) Connections between layer 4B of area 17 and thick cytochrome oxidase stripes of area 18 in the squirrel monkey. J. Neurosci. 7: 3371-3377.

Lund, J. S. (1973) Organization of neurons in the visual cortex, area 17, of the monkey (Macaca mulatta). J. Comp. Neurol. 147: 455496.

Lund, J. S., and R. G. Boothe (1975) Interlaminar connections and pyramidal neuron organization in the visual cortex, area 17 , of the macaque monkey. J. Comp. Neurol. 159: 305-334.

Matsubara, J., M. Cynader, N. V. Swindale, and M. P. Stryker (1986) Intrinsic projections within visual cortex: Evidence for orientationspecific local connections. Proc. Natl. Acad. Sci. USA 82: 935-939.

Maunsell, J. H. R. (1987) Physiological evidence for two visual subsystems. In Matters of Intelligence: Conceptual Structure in Cognitive Neuroscience, L. M. Vaina, ed., Reidel, Dordrecht.

Orbach, H. S., L. B. Cohen, and A. Grinvald (1985) Optical mapping of electrical activity in rat somatosensory and visual cortex. J. Neurosci. 5: 1886-1895.

Poggio, G. F., and B. Fischer (1977) Binocular interaction and depth sensitivity of striate and prestriate cortical neurons of behaving rhesus monkeys. J. Neurophysiol. 40: 1392-1405.

Schiller, P. H., B. L. Finlay, and S. L. Volman (1976) Quantitative studies of single-cell properties in monkey striate cortex. I. Spatio- temporal organization of receptive fields. J. Neurophysiol. 39: 12881319.

Schoppmann, A., and M. P. Stryker (1981) Physiological evidence that the 2-deoxyglucose method reveals orientation columns in cat visual cortex. Nature 293: 574-576.

Shipp, S., and S. M. Zeki (1985) Segregation of pathways leading from area V 2 to areas V4 and V 5 of macaque monkey visual cortex. Nature 315: 322-325.

Silverman, M. S., and R. B. H. Tootell (1987) Modified technique for cytochrome oxidase histochemistry: Increased staining intensity and compatibility with 2-deoxyglucose autoradiography. J. Neurosci. Methods 19: 1-10.

Sperling, H. G., M. L. J. Crawford, and S. Espinoza (1978) Threshold spectral sensitivity of single neurons in the lateral geniculate nucleus of performing monkeys. Mod. Prob. Ophthalmol. 19: 2-18.

Thorell, L. G. (1980) Color in Form Vision. Ph.D. Dissertation, University of California, Berkeley.

Thorell, L. G., R. L. De Valois, and D. G. Albrecht (1984) Spatial mapping of monkey $\mathrm{V} 1$ cells with pure color and luminance stimuli. Vision Res. 24: 751-769.

Tootell, R. B. H., and Blasdel, G. G. (1987) In vivo demonstration of presumptive blob and interblob regions by manipulation of stimulus spatial frequency in macaque striate cortex. Soc. Neurosci. Abstr. 13: 2.

Tootell, R. B. H., and M. S. Silverman (1985) Two methods for flatmounting cortical tissue. J. Neurosci. Methods 15: 177-190.

Tootell, R. B. H., M. S. Silverman, E. Switkes, and R. L. De Valois (1982) Deoxyglucose analysis of retinotopic organization in primate striate cortex. Science 218: 902-904.

Tootell, R. B. H., M. S. Silverman, R. L. De Valois, and G. H. Jacobs (1983) Functional organization of the second cortical visual area of primates. Science 220:737-739.

Tootell, R. B. H., S. L. Hamilton, and M. S. Silverman (1985) Topography of cytochrome oxidase activity in owl monkey cortex. J. Neurosci. 5: 2786-2800.

Tootell, R. B. H., S. L. Hamilton, M. S. Silverman, and E. Switkes (1988a) Functional anatomy of macaque striate cortex. I. Ocular dominance, baseline conditions, and binocular interactions. J. Neurosci. $8: 1500-1530$.

Tootell, R. B. H. F. Switkes, M. S. Silverman, and S. L. Hamilton (1988b) Functional anatomy of macaque striate cortex. II. Retinotopic organization. J. Neurosci. 8: 1531-1568.

Tootell, R. B. H., M. S. Silverman, S. L. Hamilton, R. L. De Valois, and E. Switkes (1988c) Functional anatomy of macaque striate cortex. III. Color. J. Neurosci. 8: 1569-1593.

Tootell, R. B. H., S. L. Hamilton, and E. Switkes (1988d) Functional anatomy of macaque striate cortex. IV. Contrast and magno/parvo streams. J. Neurosci. 8: 1594-1609.

Tootell, R. B. H., M. S. Silverman, S. L. Hamilton, E. Switkes, and R. L. De Valois (1988e) Functional anatomy of macaque striate cortex. V. Spatial frequency. J. Neurosci. 8: 1610-1624.

Tootell, R. B. H., R. T. Born, and S. L. Hamilton (1988f) Studies of primate visual cortex using a double-label DG technique and color autoradiography. Soc. Neurosci. Abstr. 14: 897.

Ts'o, D. Y., R. D. Frostig, E. E. Licke, and A. Grinvald (1988) Functional organization of visual area 18 of macaque as revealed by optical imaging of activity-dependent intrinsic signals. Soc. Neurosci. Abstr. 14: 898 .

Van Essen, D. C., and S. Zeki (1978) The topographic organization of rhesus monkey prestriate cortex. J. Physiol. (Lond). 277: 192-226.

Wong-Riley, M. T. T. (1979) Columnar cortico-cortical interconnections within the visual system of the squirrel and macaque monkeys. Brain Res. 162: 201-217.

Wong-Riley, M. T. T., and E. W. Carroll (1984) Quantitative light and electron microscopic analysis of cytochrome oxidase-rich zones in VII prestriate cortex of the squirrel monkey. J. Comp. Neurol. 222: $18-37$.

Zeki, S. M. (1978) Uniformity and diversity of structure and function in rhesus monkey prestriate cortex. J. Physiol. (Lond.) 277: 273-290. 